\title{
Identification of BHB splicing motifs in intron-containing tRNAs from 18 archaea: evolutionary implications
}

\author{
CHRISTIAN MARCK ${ }^{1}$ and HENRI GROSJEAN ${ }^{2}$ \\ ${ }^{1}$ Service de Biochimie et de Génétique Moléculaire, CEA/Saclay, 91191 Gif-sur-Yvette, France \\ ${ }^{2}$ Laboratoire d'Enzymologie et Biochimie Structurales, Centre National de la Recherche Scientifique, 91198 Gif-sur-Yvette, France
}

\begin{abstract}
Most introns of archaeal tRNA genes (tDNAs) are located in the anticodon loop, between nucleotides 37 and 38 , the unique location of their eukaryotic counterparts. However, in several Archaea, mostly in Crenarchaeota, introns have been found at many other positions of the tDNAs. In the present work, we revisit and extend all previous findings concerning the identification, exact location, size, and possible fit to the proposed bulge-helix-bulge structural motif $\left(B H B\right.$, now renamed $\left.h B H B h^{\prime}\right)$ of the sequences spanning intron-exon junctions in intron-containing tRNAs of 18 archaea. A total of 103 introns were found located at the usual position $37 / 38$ and 33 introns at 14 other different positions, that is, in the anticodon stem and loop, in the $\mathrm{D}$-and T-loops, in the $\mathrm{V}$-arm, or in the amino acid arm. For introns located at 37/38 and elsewhere in the pre-tRNA, canonical $\boldsymbol{h} B \boldsymbol{H} \boldsymbol{B} \boldsymbol{h}^{\prime}$ motifs were not always found. Instead, a relaxed $\boldsymbol{h} B \boldsymbol{H}$ or $\boldsymbol{H B} \boldsymbol{h}^{\prime}$ motif including the constant central 4-bp helix $H$ flanked by one helix ( $h$ or $h^{\prime}$ ) on either side generating only one bulge could be disclosed. Also, for introns located elsewhere than at position 37/38, the $h B H B h^{\prime}$ (or $H B h^{\prime}$ ) structure competes with the three-dimensional structure of the mature tRNA, attesting to important structural rearrangements during the complex multistep maturation-splicing processes. A homotetramer-type of splicing endonuclease (like in all Crenarchaeota) instead of a homodimeric-type of enzyme (as in most Euryarchaeota) appears to best fit the requirement for splicing introns at relaxed $h B H$ or $H B h^{\prime}$ motifs, and may represent the most primitive form of this enzyme.
\end{abstract}

Keywords: Archaea; Eukarya; evolution; intron; RNA processing; small RNA; splicing endonuclease

\section{INTRODUCTION}

Previously, we extracted, analyzed, and compared the nucleotide sequences of over 4000 tRNA genes (tDNAs) from 50 fully sequenced genomes, of which 13 originated from Archaea (Marck and Grosjean 2002). From the various features analyzed, Archaea appear as an intermediate domain between Eukarya and Bacteria, sharing many properties with either or both domains depending upon the

Reprint requests to: Christian Marck, Service de Biochimie et de Génétique Moléculaire, Bât. 144, CEA/Saclay, F-91191 Gif-sur-Yvette CEDEX, France; e-mail: christian.marck@cea.fr; fax: 33 (0)16908 4712.

Abbreviations: 2D, two-dimensional; 3D, three-dimensional; A:C, A:G, $\mathrm{C}: \mathrm{T}$, and so on, mismatched base pairs; tDNA, tRNA gene; BHB, bulgehelix-bulge; $\mathbf{h B H B h}$ ', a canonical BHB splicing motif with the two outer helices having at least two Watson-Crick base pairs; $\mathbf{h}$, the helix closing the 3-nt bulge on the exonic side; $\mathbf{h}^{\prime}$, the helix closing the 3 -nt bulge on the intronic side; $\mathbf{h B H}$, a relaxed $\mathrm{BHB}$ splicing motif in which the $\mathbf{h}^{\prime}$ intronic helix has less than 2 bp; $\mathbf{H B h}^{\prime}$, a relaxed BHB splicing motif in which the $\mathbf{h}$ exonic helix has less than 2 bp. The universal, conventional numbering system for tRNA positions corresponds to that adopted in the tRNA sequence bank (Sprinzl et al. 1998).

Article and publication are at http://www.rnajournal.org/cgi/doi/ 10.1261/rna.5132503.
tDNA property considered. However, a unique feature of archaeal tDNAs is their ability to occasionally host introns at various locations other than the usual position within the anticodon loop (between nt 37 and 38) of the tRNA precursors. Although these unusually located introns in archaeal pre-tRNAs had already been observed in 1987, either within or $5^{\prime}$ to the anticodon (Wich et al. 1987), identification of an enzyme-dependent spliceable intron outside the anticodon loop of pre-tRNA was made later by the group of Garrett. They identified an unusually located 18nt-long intron in the variable loop of the precursor tRNAGly (CCC) of the extreme thermophile Thermophilum pendens (Kjems et al. 1989b). In this pre-tRNA, the exonintron boundaries were able to fold into a structure similar but not identical to the characteristic bulge-helix-bulge (BHB) usually found in the intron-exon boundaries located at position 37/38 of pre-tRNAs (Kaine et al. 1983; Daniels et al. 1985; Daniels and Dougas 1986; Kaine 1987; Datta et al. 1989; Thompson et al. 1989; Thompson and Daniels 1990; Kleman-Leyer et al. 1997) and in the long Watson-Crick base-paired stems of a few rRNA precursors of hyperthermophilic Archaea (Kjems and Garrett 1988; Dalgaard and 
Garrett 1992; Burggraf et al. 1993; Durovic and Dennis 1994; Lykke-Andersen and Garrett 1994; Dennis et al. 1998; Itoh et al. 1998; Nomura et al. 1998; Ciammaruconi and Londei 2001). More recently, introns were discovered in the anticodon loop between nt 32 and 33 in the pre-tRNA-Pro (GGG) of Methanobacterium thermoautotrophicum (Smith et al. 1997), in the D-loop and anticodon stem of several tDNAs of Sulfolobus solfataricus (She et al. 2001), and in many other locations in tDNAs of Pyrobaculum aerophilum (Fitz-Gibbon et al. 2002).

The canonical BHB structural motif usually found at the exon-intron boundaries within the anticodon loop of archaeal pre-tRNAs consists of a 4-bp helix flanked on each $3^{\prime}$ side with $3 \mathrm{nt}$ in a bulge and was demonstrated to be the conformational structure most easily recognized by a unique archaeal splicing machinery (Thompson and Daniels 1988, 1990; Kleman-Leyer et al. 1997) that is supposed to work on both tRNA and rRNA precursors (Kjems et al. 1989a; Kjems and Garrett 1991; Lykke-Andersen et al. 1997; Tang et al. 2002; Salgia et al. 2003). A similar predicted BHB motif at intron-exon boundaries of messenger RNA precursors originating from three crenarchaeal species of thermophilic or hyperthermophilic archaea has been recently described, suggesting that the splicing of these premRNAs probably depends on the same machinery as the one for archaeal pre-tRNAs and pre-rRNAs (Watanabe et al. 2002).

In the present work, we revisit and extend our earlier observations concerning the identification, exact location, size, and fit to the canonical BHB motif of all introns present in tDNAs of the 18 archaeal complete genomes available to date (September 2003). Beside the fact that we identified a novel intron position in the amino acid stem of several tDNAs of $P$. aerophilum, we noticed, together with others, that the nucleotide sequences of a few introns located between nt 37 and 38 and at other positions of the pre-tRNA sometimes exibit a more relaxed $\mathrm{BHB}$ motif than the strict $\mathrm{BHB}$ rule as defined earlier for introns located at the canonical position $37 / 38$ in pre-tRNAs (see references above). Examples of BHB two-dimensional (2D) structures presented in this work are taken from pre-tRNAs of two especially interesting archaea: $P$. aerophilum, a nitrate-reducing hyperthermophilic crenarchaeon $\left(T o p t=100^{\circ} \mathrm{C}\right)$ the tDNAs of which contain the largest number of unusually located introns (19 altogether) and Sulfolobus tokodaii, an aerobic thermophilic crenarchaeon $\left(T o p t=80^{\circ} \mathrm{C}\right)$ that contains a large number of introns exhibiting relaxed $\mathrm{BHB}$ motifs (15 altogether). The structural characteristics of these unusual archaeal introns in pre-tRNAs are discussed in relation to those described in pre-rRNAs (Lykke-Andersen et al. 1997) and pre-mRNAs of Archaea (Watanabe et al. 2002). Also, a correlation between the oligomeric structure of the splicing endonuclease and the type of $\mathrm{BHB}$ motif suggests a possible evolutionary scenario for the emergence and the evolution of the splicing machinery within the three domains of life. The 800 aligned tDNAs sequences (with introns detailed) extracted from the 18 archaeal genomes are available upon request as Supplementary Material (send an e-mail message containing the keyword "tDNAs_18_Genomes" to christian.marck@cea.fr).

\section{RESULTS}

\section{Few archaea host tDNA introns at location others than "37/38"}

Without exception, the archaea of which the complete genome sequence was analyzed contain introns in their tRNA genes (tDNA; Table 1; see also Marck and Grosjean 2002). However, most of them contain only 2-5 intron-bearing tDNAs out of a population of 34-47 tDNAs per genome. In all cases but one, intron-containing tDNAs are present as single-copy genes [two copies of the intron-containing tDNA-Glu (TTC) are present in Methanopyrus kandleri]. These introns are most frequently found at canonical position $37 / 38$ in the anticodon loop (see below), the unique location in their eukaryotic counterparts. Of the 18 archaea considered, only six contain tDNAs harboring introns at position others than 37/38. These are the four crenarchaeota: P. aerophilum, Aeropyrum pernix, S. solfataricus, and $S$. tokodaii, and the two (out of 14) euryarchaeota: $M$. kandleri and M. thermoautotrophicum.

Altogether, we found 136 introns among the 800 tDNAs analyzed, of which $103(75 \%)$ are located at position 37/38 and only $33(25 \%)$ are distributed among 14 different positions within the pre-tRNA molecules: in the anticodon branch, in the variable arm, and in the D- and T-loops as well as in the amino acid arm (Fig. 1). In six cases, two introns are found within the same precursor tRNA (Table 1; indicated by $1 / 2$ and $2 / 2$ in Fig. 1). Among all archaea examined, the crenarchaeon $P$. aerophilum contains the largest number of tDNA introns (26 altogether), the largest proportion of introns located at noncanonical positions (19 cases) and the largest number of two introns within the same tDNA (three cases; Table 1). The three closely related species Pyrococcus abyssi, Pyrococcus horikoschii, and Pyrococcus furiosus contain tDNAs harboring introns only at the usual position $37 / 38$ of the anticodon loop. Thus, not all thermophilic or hyperthermophilic archaea contain introns at nonconventional locations.

\section{The majority of introns at " $37 / 38$ " obey the canonical BHB splicing motif}

Figure 2A lists a selection of 30 sequences of exon-intron junctions out of a total of 103 identified at position 37/38 in the 800 archaeal tDNAs analyzed (a complete list is available as Supplementary Material). The most often encountered $37 / 38$ BHB splicing motif (67 out of 103; see Table 1 ) is the one that was initially defined by the group of Daniels and Garrett (Kjems et al. 1989a; Thompson and Daniels 1990). 


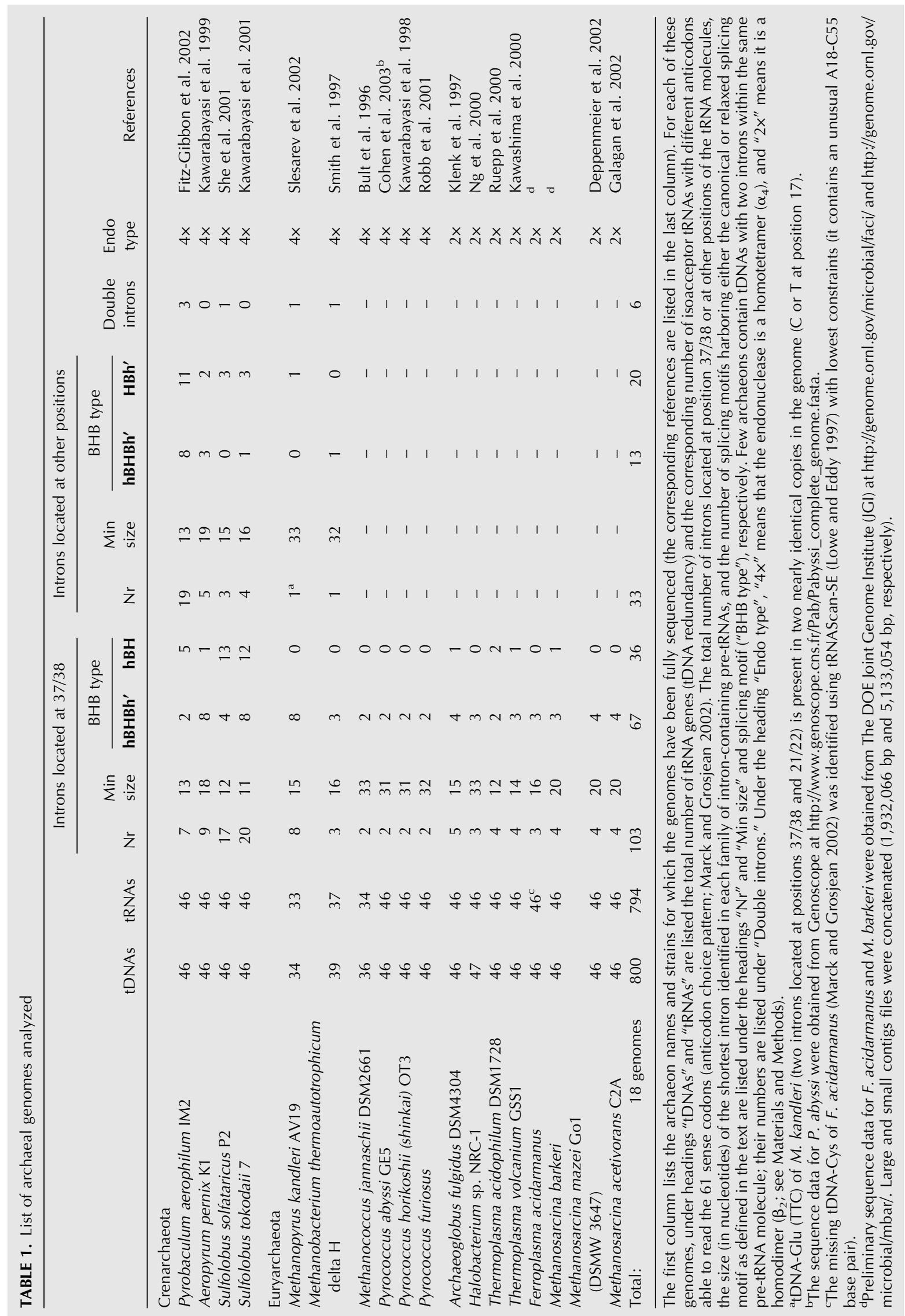




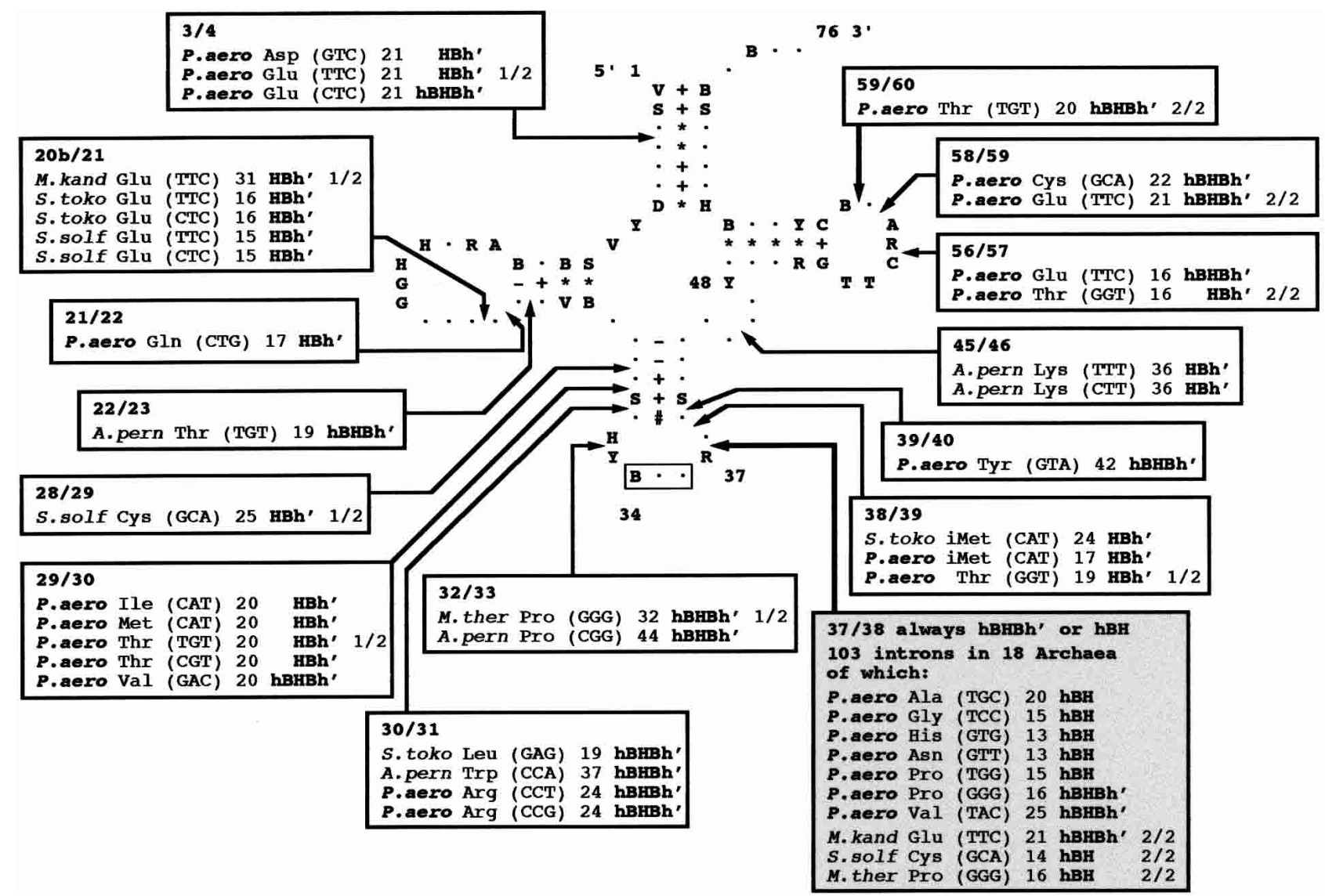

FIGURE 1. Location, size, and BHB type of introns in the tRNA genes of 18 Archaea. The schematic cloverleaf sequence corresponds to the consensus sequence of all the tDNAs (257 altogether) from the six archaea that contain introns at positions other than 37/38 (33 altogether; see Table 1). The conventional IUB/IUPAC degenerate DNA alphabet (Cornish-Bowden 1985) is used in this and the following figures: $\mathbf{R}$ (purine), A or G; Y (pyrimidine), C or T; S (strong), G or C; B (not A), C, G, or T; D (not C), A, G, or T; H (not G), A, C, or T; V (not T), A, C, or G; $\mathbf{N}$ (any), A, C, G, or T. Key to base-pairing consensus is: (plus), Watson-Crick base pairing only; (asterisk), Watson-Crick or G-T/T-G pairings; (number sign), Watson-Crick pairing or mismatch; (minus), Watson-Crick pairing or G-T/T-G pairings or mismatches. Arrows show the positions where introns are located. Their exact locations are also indicated in the first line of each box (for example, 3/4 means that the intron is located between nt 3 and nt 4 . Every intron is indicated with the abbreviated name of the species [with those of $P$. aerophilum ( $P$. aero) highlighted in bold], the tDNA type (amino acid), the length of the intron, and its type of splicing motif (hBHBh', $\mathbf{h B H}$, or $\mathbf{H B h}$; see text). The $1 / 2$ and $2 / 2$ indications refer to the six cases of two introns within the same tDNA. In three of these six cases, one of the introns is located at $37 / 38$ and is indicated accordingly. All tDNAs bearing introns in the 18 archaeons examined are essential and single-copy genes, except the case of tDNA-Glu (TTC) of M. kandleri (two copies; see also Table 1).

Figure 3A,B,C illustrates three examples of 2D structure of BHB splicing motifs in three selected archaeal pretRNAs. In Figure 3A, the central element is a 4-bp helix $\mathbf{H}$ (gray background, with its two antiparallel strands designated $\mathbf{H} 1$ and $\mathbf{H} 2$ ) flanked on either side but on different strands with a 3-nt bulge, B1 and B2. Beyond both bulges, two other double-stranded helices, $\mathbf{h}$ and $\mathbf{h}^{\prime}$ (boxed in Fig. $3 \mathrm{~A})$ are present with their antiparallel strands designated $\mathbf{h} \mathbf{1}$ and $\mathbf{h} \mathbf{2}$, and $\mathbf{h}^{\prime} \mathbf{1}$ and $\mathbf{h}^{\prime} \mathbf{2}$, respectively. The whole structure (the three double helices $\mathbf{h}, \mathbf{H}, \mathbf{h}^{\prime}$, and the two bulges $\mathbf{B} \mathbf{1}$ and B2) has a twofold symmetry axis located in the middle of the $\mathbf{H}$ helix, which is further stabilized by costacking with the rest of the pre-tRNA structure (the D-arms, located at the upper side of helix $\mathbf{H}$ in Fig. 3A,B,C) and by the closing intron loop at the opposite (bottom) side. We designate the two symmetrically oriented halves of the complete canoni- cal BHB structure (Fig. 3A) as hHB for the upper part (where $\mathbf{h}$ is the exonic helix; upward arrow) and $\mathbf{H} \mathbf{B h}^{\prime}$ for the lower part (where $\mathbf{h}^{\prime}$ is the intronic helix; downward arrow), the whole symmetric structure being designated as hBHBh' $^{\prime}$. Notice that only introns at least $14 \mathrm{nt}$ long will allow the formation of a minimal 2-bp helix $\mathbf{h}^{\prime}$ (with $3 \mathrm{nt}$ allowed for the loop). Also the anticodon (boxed in Fig. $3 \mathrm{~A}, \mathrm{~B}, \mathrm{C})$ occupies the two last positions of strand $\mathbf{H} \mathbf{1}$, the forward strand of double helix $\mathbf{H}$, and the first position of bulge B1. After cleavage by the archaeal endonuclease and subsequent enzymatic ligation of the two exonic ends and circularization of the intron, two hairpin loops, one for the seven-membered tRNA anticodon loop and the other for the circularized intron, will be generated (Salgia et al. 2003 and references therein).

However, not all $37 / 38$ intron-exon junctions obey this 


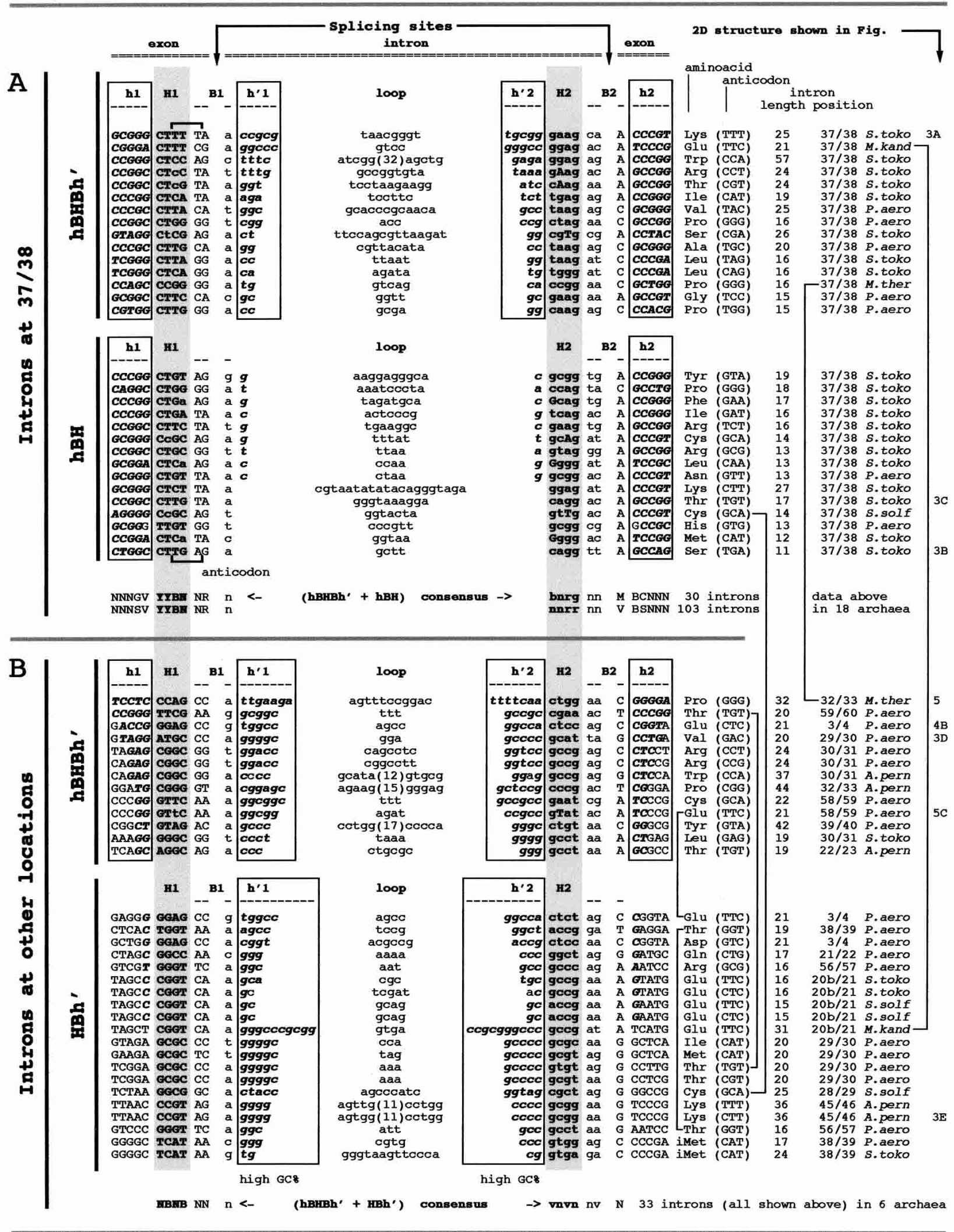

C

ABMS NN $\mathrm{n}<-$ (hBHBh' + hBH + HBh') consensus $\rightarrow$ nnvn $\mathrm{nn} N$ all 136 introns in all 18 archaea 
canonical hBHBh' splicing motif. Among introns located at position $37 / 38$, some are, in fact, too short to allow for the formation of an intronic double helix $\mathbf{h}^{\prime}$. The shortest $37 / 38$ intron that we found, among the 18 archaeon sequences examined, is that of pre-tRNA-Ser (UGA) of S. tokodaii (11 bases, listed in Fig. 2A and shown in Fig. 3B). In this case, due to the quasi absence of the intronic helix $\mathbf{h}^{\prime}$, the 3-nt bulge $\mathbf{B} 1$ cannot be generated. Only the exonic part of the BHB structure (indicated by the arrowed $\mathbf{~ h B H}$ in Fig. $3 \mathrm{~B}$ ) is present and we refer to these variants of the BHB motif as $\mathbf{h B H}$ to indicate the lack of $\mathbf{h}^{\prime}$ helix. Following $\mathrm{Li}$ and Abelson (2000), we consider an intron located at position 37/38 to be a $\mathbf{h B H}$ type (and not a $\mathbf{h B H B h}$ ') if the $\mathbf{h}^{\prime}$ helix within the intron is shorter than $2 \mathrm{bp}$. Figure $3 \mathrm{C}$ shows another example of an hBH-type intron in the pre-tRNA-Thr (UGU) of S. tokodaii. Here, the intron is longer (17 nt), but the $\mathbf{h}^{\prime}$ helix cannot be formed due to the lack of complementary bases. Therefore, the canonical $\mathbf{h B H B h}$ ' splicing motif in intron-exon junctions of archaeal pre-tRNAs can obviously be relaxed into a simpler structure, $\mathbf{h B H}$, where only two helices (h and $\mathbf{H}$ ) and one bulge (B2) are present (Fig. 3B,C).

A consensus sequence has been reported for the $\mathbf{h B H B h}{ }^{\prime}$ motif as part of the anticodon loop within the $\mathbf{H}$ helix (Kleman-Leyer et al. 1997). However, this consensus sequence is probably not essential for the endonuclease recognition because it results from the quasi-strict conservation of pyrimidine at position 32 (first nucleotide in the $\mathbf{H 1}$ strand), uridine (but one $\mathrm{C}$ ) at position 33 (second nucleotide) and purine at position 37 (second nucleotide of B1) in the anticodon loops of all archaeal tRNAs (see consensus in Fig. 2A; see also Table I in Marck and Grosjean 2002). In the 103 introns located at position 37/38, a few mispairings were observed in helix H: A:C (11 times), A:G (five times), C:T (three times), T:T (twice; Fig. 2A; Supplementary Material).

\section{Relaxed splicing motifs predominate in intron-exon junctions at positions other than $37 / 38$}

All cases of introns identified at positions others than 37/38 (33 cases among 14 different positions in 800 tDNAs) are listed in Figure 2B (see also Fig. 1). As for the case of 37/38 introns, the intron-exon junction can fold either into the

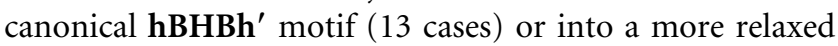
motif, $\mathbf{H B h}^{\prime}$ (20 cases), with an $\mathbf{h}^{\prime}$ helix of at least 3 bp for the hyperthermophilic crenoarchaeon $P$. aerophilum and 2 bp for the thermophilic ones (S. sulfolobus and S. tokodaii). Figure 3D illustrates the case of the pre-tRNA-Val (GAC) of $P$. aerophilum, in which the intron located at position 29/30 of the anticodon stem can be folded into the canonical hBHBh' motif, and Figure 3E illustrates the case of pretRNA-Lys (CUU) of A. pernix, in which the intron located at position $45 / 46$ of the $\mathrm{V}$-arm cannot fold into a perfect canonical splicing motif. However, it can fold into a relaxed $\mathbf{H B h}^{\prime}$ motif. In this latter case, the intronic (rightmost) part of the intron-exon junction can be folded into a hairpin structure with $4 \mathrm{bp}$ in the $\mathbf{h}^{\prime}$ helix and a large loop, whereas the exonic (leftmost) part remains unpaired (dotted box).

In introns located at positions other than 37/38, apart from Watson-Crick base pairing in $\mathbf{H}$ and $\mathbf{h}^{\prime}$ helices and a strong preference for GC base pairs in $\mathbf{h}^{\prime}$, no strict nucleotide conservation is observed (a single case of T:T mispairing was observed in the pre-tRNA-Glu (TTC) of P. aerophilum; see in Fig. 2B). However, we cannot exclude that selected nucleotide sequences, like the GNRA (Heus and Pardi 1991) or UNCG (Cheong et al. 1990) sequences within the intronic part (for review, see Michel and Costa 1998) may be important for stabilizing the loop of short hairpins. No intron shorter than 17 or $15 \mathrm{nt}$ (for hyperthermophiles and thermophiles, respectively) is found for the $\mathbf{H B h}^{\prime}$ motif compared to $11 \mathrm{nt}$ in $\mathbf{h B H}$ introns, probably because of the requirement for a sufficiently stable $\mathbf{h}^{\prime}$

FIGURE 2. Intron-exon sequences in selected archaeal tDNAs sorted out as a function of the type of their splicing motifs. The details of the sequences of all introns presented in Figure 1 are given here. Additionally, all introns (any type) of $S$. tokodaii are illustrated. (A) Only 30 sequences of intron-exon junctions (out of a total of 103 sequences in the 800 tDNAs analyzed; see Supplementary Material) corresponding to intron located at position $37 / 38$ are listed. The sequences shown extend from base 27 to base 43 of the anticodon arm. Uppercase letters correspond to exonic sequence and the lowercase letters to the intronic sequence. The bases in the $\mathbf{H}$ helix (under headings $\mathbf{H} \mathbf{1}$ and $\mathbf{H} 2$; gray background) are in bold; complementary bases in $\mathbf{h}$ and $\mathbf{h}^{\prime}$ helices (strands $\mathbf{h} \mathbf{1}, \mathbf{h}^{\prime} \mathbf{1}, \mathbf{h}^{\prime} \mathbf{2}$ and $\mathbf{h} \mathbf{2}$ ) are in bold italics. Mismatches in $\mathbf{H}$ helix are shown with inverted case: lowercase in $\mathbf{H 1}$ (exon) and uppercase in $\mathbf{H} \mathbf{2}$ (intron). Those that exhibit an intronic $\mathbf{h}^{\prime}$ helix of two or more base pairs are designated as canonical $\mathbf{h B H B h}$, and those with an intronic $\mathbf{h}^{\prime}$ helix shorter than $2 \mathrm{bp}$ as relaxed $\mathbf{h B H}$ (see examples of $2 \mathrm{D}$ structures in Fig. 3 ). Notice that, for the $\mathbf{h B H B h}^{\prime}$ type, all elements of the splicing motif can be defined (h1, H1, B1, h' $\mathbf{1}, \mathbf{l o o p}, \mathbf{h} \mathbf{2}, \mathbf{H} 2, \mathbf{B} 2$, and h2) whereas for the hBH type, only h1, $\mathbf{h} 2, \mathbf{H} 1, \mathbf{H} 2$, and $\mathbf{B} 2$ can be defined; the intronic $\mathbf{h}^{\prime} \mathbf{1}, \mathbf{h}^{\prime} \mathbf{2}$, and $\mathbf{B} \mathbf{1}$ elements cannot. At the bottom of $A$, is the consensus sequence of the splicing motif corresponding to the 30 sequences shown here or in all the 103 sequences (see Supplementary Material). Mismatched bases were not taken into account for consensus computation. For the degenerate alphabet used, see legend to Figure 1 . (B) The 33 sequences of intron-exon junctions corresponding to introns located at positions other than $37 / 38$ in the 800 tDNAs analyzed are listed as a function of the decreasing size of their $\mathbf{h}$ helix (exonic). The sequences shown extend from five bases upstream of the $5^{\prime}$ end of strand $\mathbf{H} \mathbf{1}$ down to five bases downstream from the $3{ }^{\prime}$ base of the intron. The same lettering conventions as above are used. Those that exhibit an h helix of two or more base pairs are designated as canonical $\mathbf{h B H B h}$, and those with an exonic $\mathbf{h}$ helix shorter than $\mathbf{2}$ bp as relaxed $\mathbf{H B h}$ '. In this latter case, only $\mathbf{H} \mathbf{1}, \mathbf{B} \mathbf{1}, \mathbf{h}^{\prime} \mathbf{1}, \mathbf{h}^{\prime} \mathbf{2}$, and $\mathbf{H} \mathbf{2}$ are present and, at variance with the above $\mathbf{h B H}$ motif, no exonic h1, h2, and $\mathbf{B} 2$ elements can be defined. Vertical linking lines at right show the six cases of two introns in the same pre-tRNA. Numbering at the extreme right of the figure corresponds to the figure numbers in which the $2 \mathrm{D}$ structures are shown. At the bottom of $B$ is the consensus sequence of the splicing motif corresponding to the 33 sequences listed in this panel. (C) generalized consensus from sequences shown in $A$ and $B$ and from all 136 introns in tDNAs of the genomes of the 18 archaeons analyzed. Mismatched bases in $\mathbf{H}$ helix were not taken into account for consensus computation. 


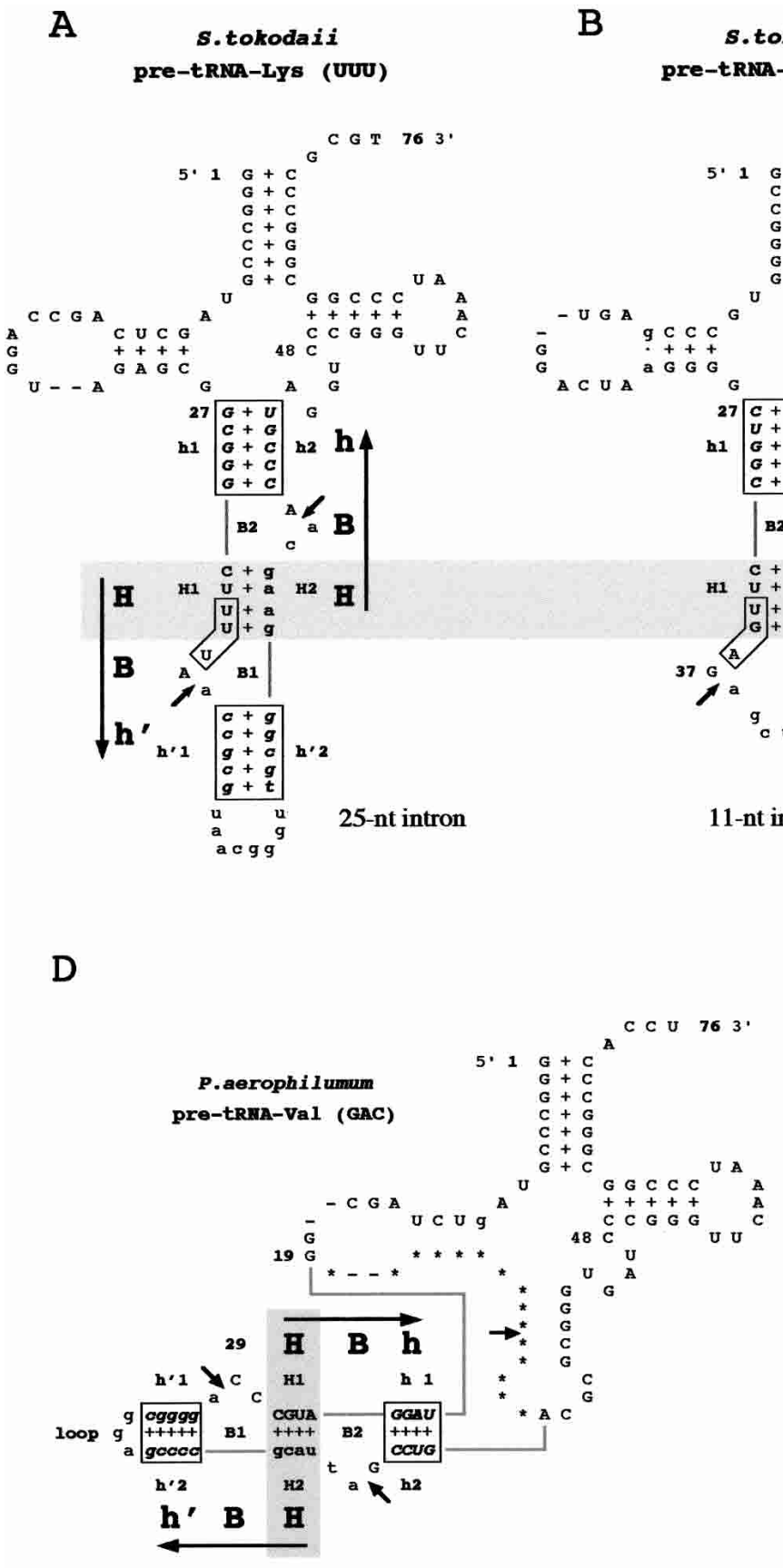

\section{$\mathbf{E}$}

intron $29 / 30$

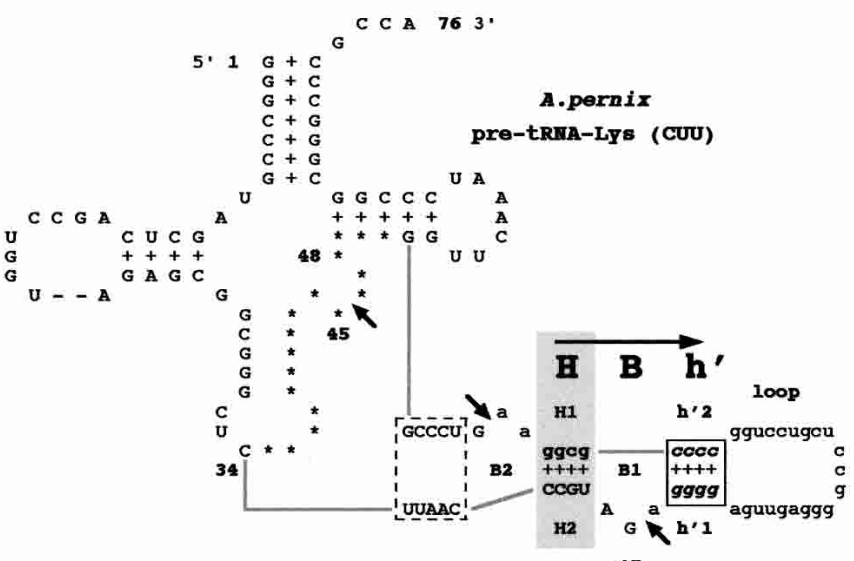

45

FIGURE 3. Examples of splicing motifs present in archaeal pre-tRNAs bearing introns at positions $37 / 38$ and others. ( $A, B, C)$ The cloverleaf sequences of three characteristic tDNAs harboring an intron between $\mathrm{nt} 37$ and 38. (D,E) Introns located at unconventional positions. Small arrows show the intron splicing position, always located two bases downstream of the $\mathbf{H} \mathbf{1}$ and $\mathbf{H} \mathbf{2}$ strands of the central $\mathbf{H}$ helix. The central 4 -bp $\mathbf{H}$ helix is highlighted with a gray background. (A) As examplified with pre-tRNA-Lys (UUU) from S. tokodaii, this panel shows the most often encountered splicing motif $\mathbf{h B H B h}$ ' (67 times over a total of 103 cases; see Supplementary Material). In this case, the exonic $\mathbf{h}$ helix (boxed) is formed by the antiparallel strands $\mathbf{h} \mathbf{1}$ and $\mathbf{h} \mathbf{2}$ of the anticodon stem. The central $\mathbf{H}$ helix (gray background) includes the first two bases of the anticodon (boxed). The third helix, $\mathbf{h}^{\prime}$ (boxed), is formed within the intron (here, $25 \mathrm{nt}$ in length). The three $\mathbf{h}, \mathbf{H}$, and $\mathbf{h}^{\prime}$ helices allow for the formation of two bulges, B1 and B2. The intron is always spliced symmetrically two bases downstream of strands $\mathbf{H 1}$ and $\mathbf{H} 2$ of helix $\mathbf{H}$. (B) In the pre-tRNA-Ser (UGA) of S. tokodaii, the intron (11 nt) located between $\mathrm{nt} 37$ and 38 is too short to allow the helix $\mathbf{h}^{\prime}$ and the bulge $\mathbf{B} 1$ to be formed. This type of relaxed splicing motif is referred to as relaxed hBH. $(C)$ In the pre-tRNA-Thr (UGU) from $S$. tokodaii, although this pre-tRNA harbors a 17-nt-long intron, the intron-exon junction can only fold into a relaxed $\mathbf{h B H}$ type of splicing motif. No complementary bases are present within the intron, preventing the formation of the intronic $\mathbf{h}^{\prime}$ helix. $(D)$ Hypothetical folding of a 20-nt intron located at position 29/30 within the pre-tRNA-Val (GAC) of P. aerophilum. Bases 20-34 are replaced within the conventional cloverleaf scheme by asterisks and are drawn in an alternative $2 \mathrm{D}$ structure that fits the splicing motif requirement. In this pre-tRNA, formation of the canonical splicing motif $\mathbf{h B H B h}$ obviously competes with the formation of the regular D- and anticodon stems. (E) Hypothetical folding of a 36-nt intron located at position 45/46 in the pre-tRNA-Lys (CUU) of A. pernix. Here, bases 35-51 of the conventional cloverleaf are replaced by asterisks and are redrawn to fit a relaxed splicing motif $\mathbf{H B h}^{\prime}$, in which a helix $\mathbf{h}$ cannot be formed because of the absence of the needed complementary bases (dotted box). This putative folding obviously competes with the formation of the regular anticodon and T-stems. 
helix. Finally, the general consensus (Fig. 2C) computed in helix $\mathbf{H}$ and the two bulges $\mathbf{B} \mathbf{1}$ and $\mathbf{B} 2$ from all introns listed in Figure 2A,B (and in Supplementary Material) shows that virtually all positions can accept any base (except in two positions where A is totally absent in helix $\mathbf{H} \mathbf{1}$ and conversely $\mathrm{Y}[\mathrm{C}$ or $\mathrm{T}]$ is totally absent in helix $\mathrm{H} 2$; B means "no $\mathrm{A}$ " and $\mathrm{V}$ means "no $\mathrm{T}$ ").

Interestingly, the $\mathbf{H B h}^{\prime}$ motif (lower part of Figs. 2B, 3E) appears to be the symmetric of the $\mathbf{h B H}$ motif (lower part of Figs. $2 \mathrm{~A}, 3 \mathrm{~B}$ ), the helix $\mathbf{h}^{\prime}$ that generates the bulge $\mathbf{B} \mathbf{1}$, being part of the intron, whereas in the case of the $\mathbf{h B H}$ type of motif, the exonic $\mathbf{h}$ helix generates the bulge $\mathbf{B 2}$. Therefore, from the enzymatic point of view, the two types of relaxed splicing motifs $\mathbf{h B H}$ (as listed in Fig. 2A) and motif

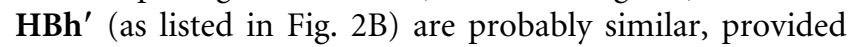
that helix $\mathbf{h}$ or $\mathbf{h}^{\prime}$ is each sufficiently stable to compensate for the lack of the missing helix $\mathbf{h}^{\prime}$ or $\mathbf{h}$ and to allow efficient recognition by the archaeal splicing endonuclease.

\section{Introns located at noncanonical positions hinder the three-dimensional (3D) structure of pre-tRNAs}

When present at position $37 / 38$ in the anticodon loop, an intron between the 3' ultimate and penultimate residues of the anticodon loop should not hinder the 2D/3D structures of the archaeal pre-tRNA. Indeed, helix $\mathbf{h}$ corresponds to the pre-tRNA anticodon stem (exon) that simply extends the coaxially stacked D-stem within the cloverleaf structure of tRNA (see Fig. 3A,B,C), whereas helix $\mathbf{h}^{\prime}$ is a hairpin terminating with a loop (intron) that will be removed and circularized after splicing, leaving a mature 7-nt anticodon loop. As a matter of fact, chemical and enzymatic probes revealed that the common tertiary structure seen in the crystal structure of yeast tRNA-Phe as model is maintained in intron-containing tRNAs and that the intron, including the splice sites, is the most exposed region of the molecule (Swerdlow and Guthrie 1984; Lee and Knapp 1985).

However, when the intron is located at any position other than $37 / 38$ within the pre-tRNAs, the situation is different. For example, the formation of the $\mathbf{h B H B h}$ ' splicing motif in pre-tRNA-Val (GAC) of $P$. aerophilum cannot be formed without complete unpairing of the D- and anticodon stems (indicated by asterisks in Fig. 3D). Similarly, the formation of a $\mathbf{H B h}^{\prime}$ type of splicing motif (with $4 \mathrm{bp}$ in the $\mathbf{h}^{\prime}$ helix and a large loop) required for removal of the 36-nt intron located between nt 45 and 46 in the V-arm of pre-tRNA-Lys (CUU) of $A$. pernix cannot be formed without unpairing the anticodon and T-stems (indicated by asterisks in Fig. $3 \mathrm{E})$. In fact, any intron located from positions $20 \mathrm{~b} / 21$ to $32 / 33$ (as indicated in Fig. 1) prevents the formation of the D- and anticodon stems, whereas those located from 38/39 to $45 / 46$ prevent the pairing of the anticodon and T-stems.

A special case concerns the intron located at position $59 / 60$ in the T-loop, like in pre-tRNA-Thr (TGT) of $P$. aerophilum (see Figs. 1, 2B), which allows the formation of an almost perfect canonical $\mathbf{h B H B h}$ ' structure. This location is equivalent to position $37 / 38$ in the anticodon loop. Indeed, in the mature tRNA, both loops have 7 bases, each stem has $5 \mathrm{bp}$, and U33 is the homolog of U55 (discussed in Marck and Grosjean 2002). However, because the T-loop interacts with the D-loop in the $3 \mathrm{D}$ architecture of the tRNA, the presence of an intron in the T-loop obviously hinders the formation of a correct $3 \mathrm{D}$ folding of the pretRNA (not shown).

\section{A novel intron location in the amino acid acceptor stem}

A very intriguing situation was found with three tDNAs from the hyperthermophilic archaeon $P$. aerophilum. The tRNA structures found for tDNA-Asp (GTC), tDNA-Glu (CTC), and tDNA-Glu (TTC) by tRNAScan-SE (Lowe and Eddy 1997; see GtRDB, http://rna.wustl.edu/GtRDB/) or by the cloverleaf tDNA search (Marck and Grosjean 2002) predict three mismatched base pairs at the end of the amino acid acceptor stem (bp 1-72, 2-71, and 3-70). Because a well-paired acceptor stem required for proper functioning of tRNA at high temperatures (above $80^{\circ} \mathrm{C}$ ) was always observed in archaeal tDNA (Marck and Grosjean 2002), we reasoned that such mispaired amino acid acceptor stems might be either posttranscriptionally edited into a perfect Watson-Crick helix (see Lavrov et al. 2000 and references therein) or resolved into a perfectly paired acceptor stem after splicing of a putative intron.

In support of this last alternative, we noticed that: (1) the transcriptional promoter TATA element upstream of each of the three tDNAs considered, which should be normally located about 20-25 bases uptream of nt 1 of the mature tRNA (Thomm and Wich 1988), is, in fact, located farther away $(59,58$, and $72 \mathrm{nt}$ away, respectively, as shown in Fig. $4 \mathrm{~A}$, in which uppercase letters represent the mature tRNA and lowercase letters the intron and flanking sequences); (2) a gcccgg sequence upstream of the tRNA gene (designated $\mathbf{h}^{\prime \prime} \mathbf{1}$, in Fig. 4A) could pair with the exonic CCGGGGC sequence (designated $\mathbf{h}^{\prime \prime 2}$ ) in positions 66-72 of the unspliced tRNA; and (3) the nucleotide sequence of this loop can be folded into a canonical HBH motif hBHBh' (Fig. 4B). If this $\mathbf{h B H B h}$ ' structure was spliced by the P. aerophilum splicing machinery, a 21-nt-long intron (box labeled "intron 3/4" in Fig. 4A) would be removed. After splicing, ligation of the two generated ends, and rearrangement of the tRNA secondary structure, a perfect Watson-Crick amino acid acceptor arm would be formed. Because these three tDNAs are highly homologous, the same sequential folding as proposed above should apply to each (Fig. 4A). However, the splicing motif in tDNA-Glu (CTC) is less perfect because only $1 \mathrm{bp}$ closes the B2 bulge (data not shown). Because the intron within the acceptor stem may hinder the recognition of precursor tRNA by the $3^{\prime}$ endonuclease and by the RNase $\mathrm{P}$, the splicing process, in this 

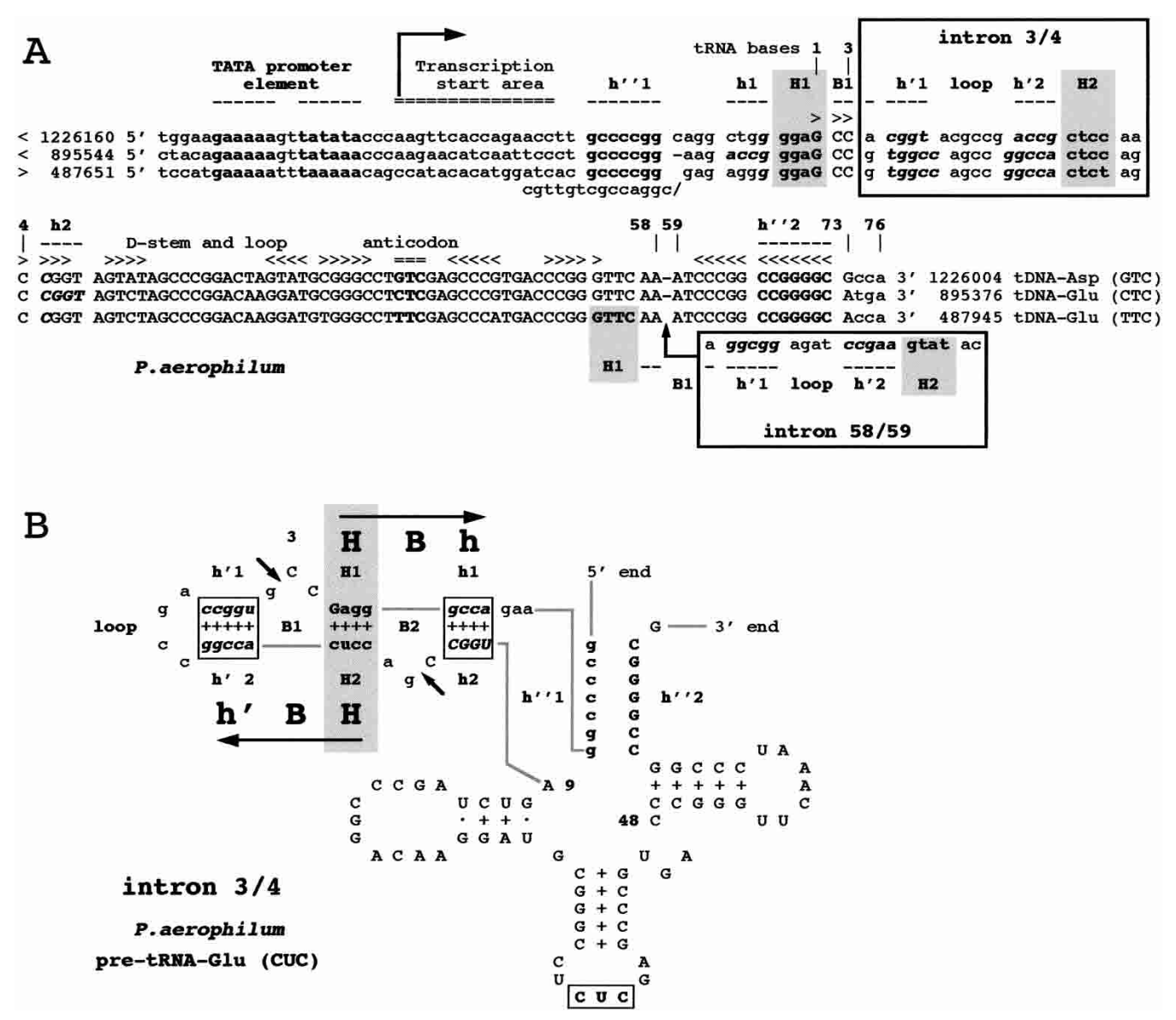

FIGURE 4. A novel intron is located at position $3 / 4$ in three tDNAs of P. aerophilum. (A) The sequences of the three tDNAs of $P$. aerophilum coding for tRNA-Asp (GUC) (upper line), tRNA-Glu (CUC) (middle line), and tRNA-Glu (UUC) (lower line) are shown. They extend from the putative upstream TATA promoter element (black bold) down to position 76 of the mature tRNA. Bases in uppercase letters belong to the mature tRNA, those in lowercase to the intron and mature tRNA flanking sequences. The bases making up the putative intron located between nt 3 and nt 4 of the amino acid acceptor stem, including the intronic helix $\mathbf{h}$, are shown in lowercase letters (within the box labeled "intron 3/4"). The sequence elements forming the canonical splicing motif $\mathbf{h B H B h}^{\prime}\left(\mathbf{h} 1, \mathbf{H} 1, \mathbf{B} 1, \mathbf{h}^{\prime} \mathbf{1}\right.$, loop, $\mathbf{h}^{\prime} \mathbf{2}, \mathbf{H} \mathbf{2}$, B2, and h2) are shown in bold (for a definition of these elements, see text and legend to Fig. 2). Bases forming the $\mathbf{H} \mathbf{1}$ and $\mathbf{H} \mathbf{2}$ strands are bold and highlighted with gray background. Bold italics are used to indicate the complementary bases forming the $\mathbf{h} \mathbf{1}$ and $\mathbf{h} \mathbf{2}$ antiparallel strands of the helix $\mathbf{h}$, and the $\mathbf{h}^{\prime} \mathbf{1}$ and $\mathbf{h}^{\prime} \mathbf{2}$ antiparallel strands of the helix h'. Among these three tDNAs, only one, tDNA-Glu (TTC), harbors a second intron located between nt 58 and 59 (box labeled "intron 58/59"). (B) Hypothetical 2D structure of pre-tRNA-Glu (CUC) including the splicing motif within the amino acid acceptor stem. The sequence shown here, including the cloverleaf-like structure, extends from 31 bases upstream from tRNA base 1 down to base 73 at the $3^{\prime}$ end of the tRNA. Plus signs are used to indicate Watson-Crick base pairing and filled circles to indicate wobble GU/UG pairings. The intron (in lowercase) is shown folded under a canonical $\mathbf{h B H B h}$ ' splicing motif, using the same lettering conventions as in $A$. The small arrows indicate the splicing positions after nt 3 and before nt 4 . The path of the nucleotidic chain is symbolized with thick gray lines. Remarkably, a peculiar gccccgg sequence is present in the $5^{\prime}$ region of the three tRNA genes, which is an exact replica of bases 1-7 of the mature tRNA. This sequence, designated $\mathbf{h}^{\prime \prime} 1$ is probably part of the pre-tRNA transcript, as it is located 20 bases $3^{\prime}$ of the TATA element. It is shown here facing the antiparallel strand of the acceptor stem (bases 66-72, designated $\mathbf{h}^{\prime \prime} 2$ ) because it is not known whether a helix between complementary strands $\mathbf{h}^{\prime \prime} 1$ and $\mathbf{h}^{\prime \prime} 2$ can form.

case, would probably have to occur before the maturation of the $3^{\prime}$ and $5^{\prime}$ ends of the pre-tRNA (Krasilnikov et al. 2003; Zahler et al. 2003; for reviews, see Hall and Brown 2001; Mörl and Marchfelder 2001; see below for other cases of mutually exclusive maturation/splicing processes). A similar obligate removal of intron before $3^{\prime}$ and $5^{\prime}$ ends maturation probably also holds for introns located at other positions (especially in the T-loop; see Fig. 1) of several pre-tRNAs.

\section{Few pre-tRNAs harbor two introns}

In addition to the intron at position 3/4 of the amino acid stem, tDNA-Glu (TTC) of P. aerophilum harbors a second intron at position 58/59 (box labeled "intron 58/59" in Fig. 4A). Here again, a canonical motif $\mathbf{h B H B h}$ ' structure can be formed (however with one mismatch in the $\mathbf{H}$ helix), without hindering the $\mathbf{H B h}^{\prime}$ structure of the intron located at positions $3 / 4$ of the amino acid arm (structure not shown). 
It is not possible to predict whether splicing of these two introns proceeds in obligate or random order.

Five other cases of two introns within the same pre-tRNA have been reported (Figs. 1, 2). Among them, only in the case of the pre-tRNA-Pro (GGG) of M. thermoautotrophicum (the first reported case of two introns within the same pre-tRNA; Smith et al. 1997), can one predict an obligate order for splicing. In this pre-tRNA, the 32-nt-long intron located at positions 32/33 (Fig. 5) obviously has to be processed before the splicing of the second intron (16 nt in length) located at the usual position $37 / 38$. Indeed, the $\mathbf{H 1}$ strand of the $\mathbf{H}$ helix of the $\mathbf{h B H B h}$ ' structure of the "second" intron (L-shaped box in the anticodon loop, Fig. 5) is interrupted between its first and second positions by the intron at positions $32 / 33$. The canonical hBHBh' motif required to splice the second intron at position $37 / 38$ can be formed only after splicing of the intron at position 32/33. This sequential removal of introns implies that there is enough plasticity of the RNA molecule within the whole anticodon stem and loop to allow major rearrangements between two successive intron splicing processes.

\section{Formation of the splicing motif also competes with other maturation processes}

Switches between mutually exclusive folding patterns of the pre-tRNA allowing distinct maturation processes to occur

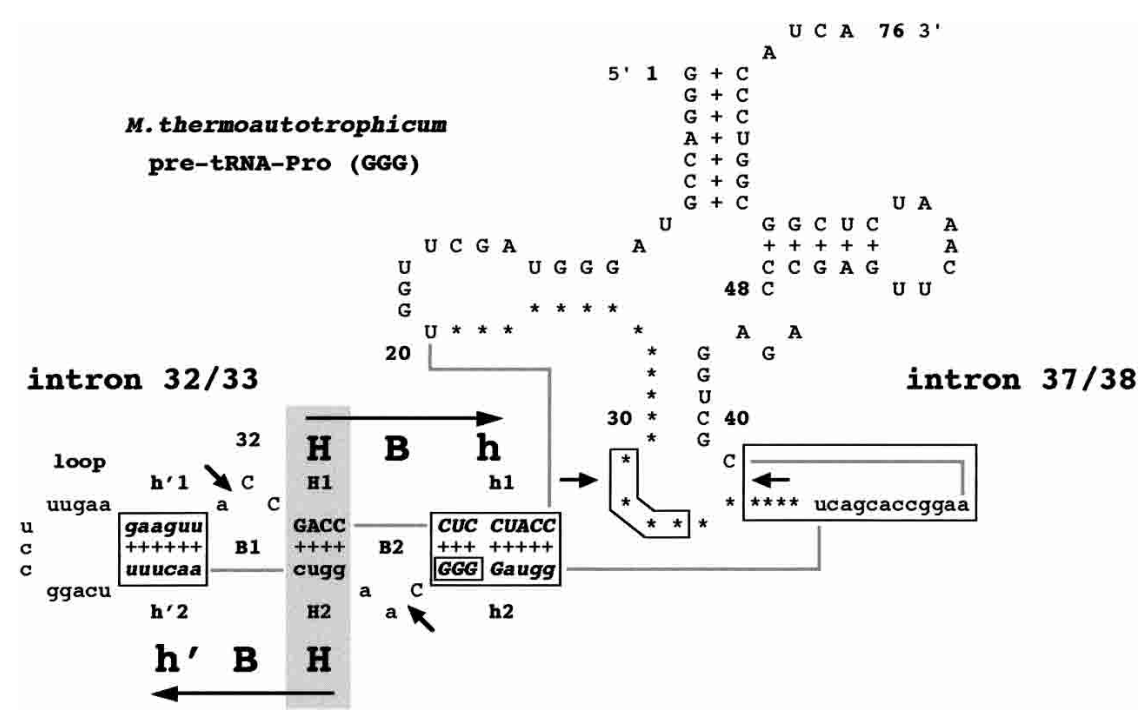

FIGURE 5. Ordered sequential intron splicing has to occur in certain pre-tRNAs harboring two introns. Pre-tRNA-Pro (GGG) of M. thermoautotrophicum contains two introns in the anticodon loop, one between nt 32 and 33 (32 nt in length) and the second one between nt 37 and 38 (16 nt in length). In the conventional cloverleaf scheme, bases 21-39 are replaced by asterisks. They are redrawn in an alternative $2 \mathrm{D}$ structure that fits the splicing motif requirement. The same lettering conventions as in Figures 1-4 are used. Only the hypothetical folding of the noncanonically located 32-nt intron is shown. In this pre-tRNA, the formation of the canonical splicing motif $\mathbf{h B H B h}$ ' (allowing the intron at position 32/33 to be spliced) obviously competes with the formation of the splicing motif corresponding to the intron located at the usual position $37 / 38$ (boxed). Only after removal of the intron at position $32 / 33$, can the intron-exon junction corresponding to intron $37 / 38$ be folded into another $\mathbf{h B H B h}{ }^{\prime}$ splicing motif (folding not shown). in an obligate order have been recently described in the case of tRNA-Trp (CCA) of Halobacterium volcanii, which harbors a long intron composed of 104 bases. In this very peculiar pre-tRNA, the intron and flanking regions not only display the hallmark $\mathbf{h B H B h}$ ' splicing motif but also a duplicate form of the hallmark $\mathrm{C} / \mathrm{D}$ boxes that guide the '-O-methylations in RNA (d'Orval et al. 2001). Based on in vitro experiments, it has been suggested that the intronic art of $H$. volcanii tRNA-Trp guides the $2^{\prime}$-O-methylation cis of both the $2^{\prime}$ hydroxyriboses at positions 34 and 39 the exon region of the precursor tRNA (Gaspin et al. that the intr generate the appropriate guide-target duplex for each independent methylation. Another structural arrangement is then required to generate the $\mathbf{h B H B h} \mathbf{H}^{\prime}$ motif recognized by the splicing endonuclease. After intron excision, the two half exons of the methylated tRNA-Trp are ligated again y a tRNA ligase (for reviews, see Bachellerie et al. 2002; mer et al. 2003). The formation of the splicing motif in above).

It is noteworthy that no intron-containing tRNAs other than archaeal pre-tRNA-Trp display RNA C/D box in their introns (d'Orval et al. 2001; Dennis et al. 2001). However, not all archaeal pre-tRNA-Trp display the characteristic $\mathrm{C} / \mathrm{D}^{\prime}$ and $\mathrm{C}^{\prime} / \mathrm{D}$ motifs. They are absent in the shorter (33 nt) intron of pre-tRNA-Trp of Methanococcus jannaschii and in the pre-tRNA-Trp of A. pernix (of which the $37-n t$ intron is located at position 30/31, not 37/38). Pre-tRNA-Trp of $P$. aerophilum does not contain any intron (Marck and Grosjean 2002). It would be interesting to know whether a $2^{\prime}$-O-methyl ribose exists at position 34 of the mature tRNA-Trp of $M$. jannaschii, A. pernix, or $P$. aerophilum. In eukaryotic cells like yeast, formation of such 2'-O-methylcytosine-34 of tRNA-Trp is catalyzed by an intron-independent enzyme (Pintard et al. 2002). It could be that $2^{\prime}-O$-methylation of C-34 in anticodon of tRNATrp as well as at many other riboses of the tRNA molecules occurs via an intron-dependent methylation machinery in some archaea (for review, see Omer et al. 2003) whereas it might be catalyzed by intron-independent tRNA modifying enzymes in other archaea (Grosjean et al. 1995; for review, see Grosjean et al. 1997; Constantinesco et al. 1999). 


\section{DISCUSSION}

Identification, comparative sequence analysis of the exonintron junctions, and careful inspection of the fit to a conserved splicing motif in all intron-containing tDNAs in a given or phylogeneticaly related organisms should allow the better definition of the minimal core motif recognized by the archaeal splicing machinery. The hallmark of intronexon boundaries in the majority of archaeal pre-tRNAs is the presence of a nucleotide sequence that can be folded into a characteristic bulge-helix-bulge, the so-called BHB motif, now renamed hBHBh'. The comparison of sequences of 136 such exon-intron boundaries in the tDNAs of 18 archaea allows us to raise the following issues.

\section{Not all archaeal intron-exon boundaries can fold into a canonical hBHBh' motif}

While examining all intron-exon junctions in the tDNAs of 18 archaea, we found that the most frequent $\mathbf{h B H B h}$ ' splicing motifs are sometimes imperfect. First, for a few introns located at 37/38 (and 59/60 in a single case), the $\mathbf{h}^{\prime}$ intronic helix is absent (less than $2 \mathrm{bp}$ ) either because the intron is too short or because no base-pairing complementarity exists between the putative strands $\mathbf{h} \mathbf{1}$ and $\mathbf{h} \mathbf{2}$. Only the exonic $\mathbf{h}$ helix is always present (Fig. 2A,B). When the intron is located at $37 / 38$, it corresponds to the anticodon stem whereas when it is located at position 59/60, it corresponds to the T-stem. Second, when the intron is located at any other place than positions $37 / 38$ and $59 / 60$, then the exonic helix $\mathbf{h}$ may be lacking, again due to the absence of base-pair complementarity. However, in such cases, a minimal $\mathbf{h}^{\prime}$ intronic helix is always present [but for one case, that of tDNA-Glu (CTC) of P. aerophilum; see Fig. 2B]. Also, a complete absence of helix $\mathbf{h}$ (no complementary bases) or $\mathbf{h}^{\prime}$ within the exon-intron boundaries of certain introncontaining pre-rRNAs has been described (see Burggraf et al. 1993; Lykke-Andersen and Garrett 1994). Altogether these observations argue against a universal perfect archaeal $\mathbf{h B H B h}^{\prime}$ structure with at least $2 \mathrm{bp}$ in helix $\mathbf{h}$ or $\mathbf{h}^{\prime}$ as predicted from structural data (Li and Abelson 2000 and see below). Instead, they reveal the existence of more relaxed motifs, $\mathbf{h B H}$ and $\mathbf{H B h}^{\prime}$ as defined in this work, that suggest less stringent structural requirements for archaeal splicing enzyme recognition, at least in some species, than has been supposed previously.

\section{Only helix $H$ and one of the two helices $h$ or $h^{\prime}$ are major determinants for splicing in certain Archaea}

The general trends that appear from the already published works together with the present thorough analysis of the nucleotide sequences of the intron-exon boundaries in 136 tDNAs from 18 complete archaeal genomes is that: (1) only the 4-bp helix $\mathbf{H}$ with no sequence preference is strictly conserved-any base change within this central helix $\mathbf{H}$ was shown to abolish in vitro splicing activity (see Thompson and Daniels 1990; Ciammaruconi and Londei 2001)-however, in a few cases, a mismatch exists (see strands $\mathbf{H} \mathbf{1}$ and H2 in gray background in Fig. 2A,B); (2) at least one helix $\mathbf{h}$ or $\mathbf{h}^{\prime}\left(\mathbf{h} \mathbf{1}, \mathbf{h} \mathbf{2}, \mathbf{h}^{\prime} \mathbf{1}\right.$, and $\mathbf{h}^{\prime} \mathbf{2}$ strands boxed in Fig. 2A,B), generating a 3-nt bulge on one of the two sides of helix $\mathbf{H}$, is always present, and their stability is probably important, as attested by their high $\mathrm{G}+\mathrm{C}$ content in pre-tRNAs, especially from the hyperthermophilic archaea; and (3) as first demonstrated by Daniels' group (Thompson and Daniels 1988; Armbruster and Daniels 1997), there is no dependence on the 3D architecture of the tRNA molecule, because archaeal introns occur in many diverse locations (14 altogether; Fig. 1), provided that the conformational plasticity of the intron-containing pre-RNA molecules allows the formation of the minimal $\mathbf{h B H}$ or $\mathbf{H B h}^{\prime}$ motif recognizable by the archaeal endonuclease (Kjems and Garrett 1991; this work). Notice that, in intron-containing prerRNAs and pre-mRNAs, at least one of the two helices $\mathbf{h}$ or $\mathbf{h}^{\prime}$ is usually very long (Garrett et al. 1991; Nomura et al. 1998; Ciammaruconi and Londei 2001; Tang et al. 2002; Watanabe et al. 2002), whereas in pre-tRNAs harboring an intron at position $37 / 38$ of the anticodon loop or at position $59 / 60$ of the T-loop, the exonic stems $\mathbf{h}$ are extended by a coaxially stacked stem (D- or T-stem, respectively). For introns located at positions other than $37 / 38$ (or 59/60), the possible influence of other parts of the folded pre-tRNA molecules on the stability of the splicing motif should not be underestimated. One main difference between cleavages at a $\mathbf{h B H B h}$ ' splicing motif or at a relaxed $\mathbf{h B H}$ or $\mathbf{H B h}^{\prime}$ variant is that, in the first case, ligation of the released fragments always generates two hairpin loops of seven residues each as in the anticodon- or T-loops of mature tRNAs (Salgia et al. 2003), whereas in the latter case, the released exons will be religated into a structure that is compatible with a subsequent folding of the RNA molecules into an appropriate functional structure (as a cloverleaf in the case of tRNAs).

Taken together, the above observations allowed us to hypothesize the need of only one stable helix $\mathbf{h}$ or $\mathbf{h}^{\prime}$, together with the central 4-bp helix $\mathbf{H}$ to generate a spliceable substrate, at least in certain Archaea, mostly Crenarchaeota (see also discussion in Kjems and Garrett 1991). Based on this assumption, the motifs $\mathbf{~} \mathbf{B H}$ and $\mathbf{H B h}^{\prime}$ appear to be the symmetric of each other. Therefore, depending on binding of the splicing endonuclease to one or the other side of this "minimalist" splicing motif, one bulge between the helix $\mathbf{H}$ and $\mathbf{h}$ or $\mathbf{h}^{\prime}$ will probably be cut first.

Detailed structural analysis of the minimal $\mathbf{h B H}$ or $\mathbf{H B h}^{\prime}$ motif, associated or not with a purified recombinant archaeal endonuclease, is not yet available. However, the RNA stem-loop structure (TAR) that contains the helical stem region separated by a 3-nt bulge, which strongly resembles the $\mathbf{H B h}^{\prime}$ structure, has been solved by NMR spectroscopy. 
The only available $\mathbf{~} B \mathrm{HBh}^{\prime}$ structure at $1.77-4.46 \AA$ average resolution determined by NMR spectroscopy is of a 38-nt RNA of a perfect $\mathbf{h B H B h}$ ' motif as found in the mesophilic euryarchaeon $H$. volcanii pre-tRNA-Trp (CCA; Diener and Moore 1998). In this structure, the conformation of the two 3-nt bulges (in which the RNA molecule is cleaved) is stabilized by stacking interactions between bulge nucleotides and bases in the adjacent helices $\mathbf{H}$ and $\mathbf{h}$ or $\mathbf{h}^{\prime}$, both bulges being presented on the same minor groove face of the central 4-bp helix $\mathbf{H}$. Interestingly, each of the two bases preceeding the cleavage site within the bulge are flipped out. However, the geometry of one of the two bulges differs somewhat from that of the other one. It is therefore possible that one of the flipped nucleotides is preferred for endonuclease attack, even in a perfect $\mathbf{h B H B \mathbf { h } ^ { \prime }}$ splicing motif.

Interestingly, the $\mathbf{h B H}$ type of splicing motif as described above has some resemblance to the splicing substrate consensus "hBh" present in most yeast pre-tRNAs harboring introns at 37/38 (for review, see Culbertson and Winey 1989; Trotta and Abelson 1999). In this latter case, the intronic helix $h$ is the same anticodon stem as for archaeal pre-tRNAs. It is flanked by a bulge (B), often larger than 3 $\mathrm{nt}$, extended by another helix $\mathrm{h}$ (the equivalent of the conserved $\mathbf{H}$ of the archaeal pre-tRNAs). In this latter helix $\mathrm{h}$, a conserved base pair between a pyrimidine of the $5^{\prime}$ exon (position 32 in tRNA) immediately following the universal $\mathrm{U}-33$ and the three bases of the anticodon and a base in the single-stranded loop of the intron (position -3, the socalled A-I base pair where A stands for anticodon and I for intron) is required for correct excision at the $3^{\prime}$ splice site (Baldi et al. 1992; for review, see Fruscoloni et al. 2001).

\section{Are the splicing motifs and oligomeric structure of the endonuclease evolutionary related?}

The "approximately" twofold symmetry of this perfect hB$\mathbf{H B h}^{\prime}$ splicing motif makes it well suited for interaction with each of the two types of RNA splicing endonuclease (RNase) EndA characterized so far: the homodimeric enzyme $\left(\beta_{2}\right)$ with a monomer size in the range 288-353 residues or the homotetrameric enzyme $\left(\alpha_{4}\right)$ with a monomer size in the range 170-187 residues (see Table 1 and Materials and Methods). From in vitro RNA footprinting experiments on the complex formed between the pure recombinant homotetrameric endonuclease $\left(\alpha_{4}\right)$ from the euryarchaeon $M$. jannaschii and a synthetic RNA substrate bearing a perfect hBHBh' splicing motif (as in crenarchaeal Desulfurococcus mobilis rRNA), it has been demonstrated that only the nucleotides outsite the minimal BHB sequence, thus including the two flanking helices $\mathbf{h}$ and $\mathbf{h}^{\prime}$ adjoining the central BHB motif, were susceptible to chemical reagents; the backbone at the two 3-nt bulges and the central 4-bp helix $\mathbf{H}$ being protected by the endonuclease (LykkeAndersen and Garrett 1997).

On the other hand, the $\alpha_{4}$-type of endonuclease of $M$. jannaschii (Li et al. 1998) has been crystallized and its 3D structure obtained at 2.3- $\AA$ resolution. This homotetramer $\left(\alpha_{4}\right)$, with small subunits of less than 200 amino acid residues, is believed to assemble through dimer intermediates; only two of the four subunits playing the role of catalytic subunits, and the two others acting as structural dimerization subunits (Lykke-Andersen and Garrett 1997; Li et al. 1998; Bujnicki and Rychlewski 2000). This type of subunit organization is present in the endonucleases of all four Crenarchaeota and in 6 out of 14 Euryarchaeota examined (Table 1; Fig. 6). More recently, the crystal structure at 2.8 $\AA$ resolution of the other type of endonuclease from $A r$ chaeoglobus fulgidus has been obtained and its 3D architecture compared with that of the $M$. jannaschii endonuclease ( $\mathrm{Li}$ and Abelson 2000). This enzyme is a homodimer $\left(\beta_{2}\right)$ with each monomer being about twice as large as the homotetrameric enzyme. Each monomer bears two homologous repeats, with the $\mathrm{C}$-terminal repeat being the most similar to the $\alpha$ subunit of the homotetrameric enzyme. This is also the part of the large subunit that has retained the catalytic function. The overall shape and subunit disposition of this homodimeric enzyme, which is found only in Euryarchaeota (eight out of 14 analyzed; Fig. 6; Table 1), is essentially the same as in the homotetrameric enzyme ( $\mathrm{Li}$ and Abelson 2000).

Docking of the hBHBh' structure of a minimalist 38-nt RNA substrate as determined by NMR spectroscopy (Diener and Moore 1998) onto the X-ray structure of the homodimeric endonuclease of $M$. jannaschii favors the idea that the binding of the two 3-nt bulges $\mathbf{B}$ is required to activate the splicing reactions and that the minimal length of the $\mathbf{h}$ and $\mathbf{h}^{\prime}$ is $2 \mathbf{n t}$ ( $\mathrm{Li}$ and Abelson 2000). These data are in agreement with the in vitro RNA footprinting experiments (Lykke-Andersen and Garrett 1997). Unfortunately, no crystal structure of the $\alpha_{4}$ type of splicing endonuclease originating from an archaeon like P. aerophilum or S. tokodaii has yet been described. Such structural information should help to better understand putative variations in the substrate structural requirements of the endonucleases from evolutionarily related organisms. Indeed, among the 18 archaeal genomes investigated, only 6 of them harbor tDNA introns located at noncanonical positions (other than 37/ 38 , and their splicing endonuclease is of the homotetrameric $\alpha_{4}$ type. Regarding the Euryarchaeota/Crenarchaeota boundary, four of them (P. aerophilum, A. pernix, $S$. sulfolobus, and $S$. tokodaii) belong to the phylum Crenarchaeota whereas the two other (M. kandleri and M. thermoautotrophicum) belong to the phylum Euryarchaeota (Fig. 6; Table 1). Among the remaining 12 archaea that do not harbor introns elsewhere than at position 37/38, some have a homotetrameric $\left(\alpha_{4}\right)$ splicing enzyme $(M$. jannashii and the three Pyrococcus) and the others have a homodimeric $\left(\beta_{2}\right)$ enzyme. No known archaeon with a dimeric enzyme has any intron located at noncanonical positions. Other archaea are known (not investigated here) that do 


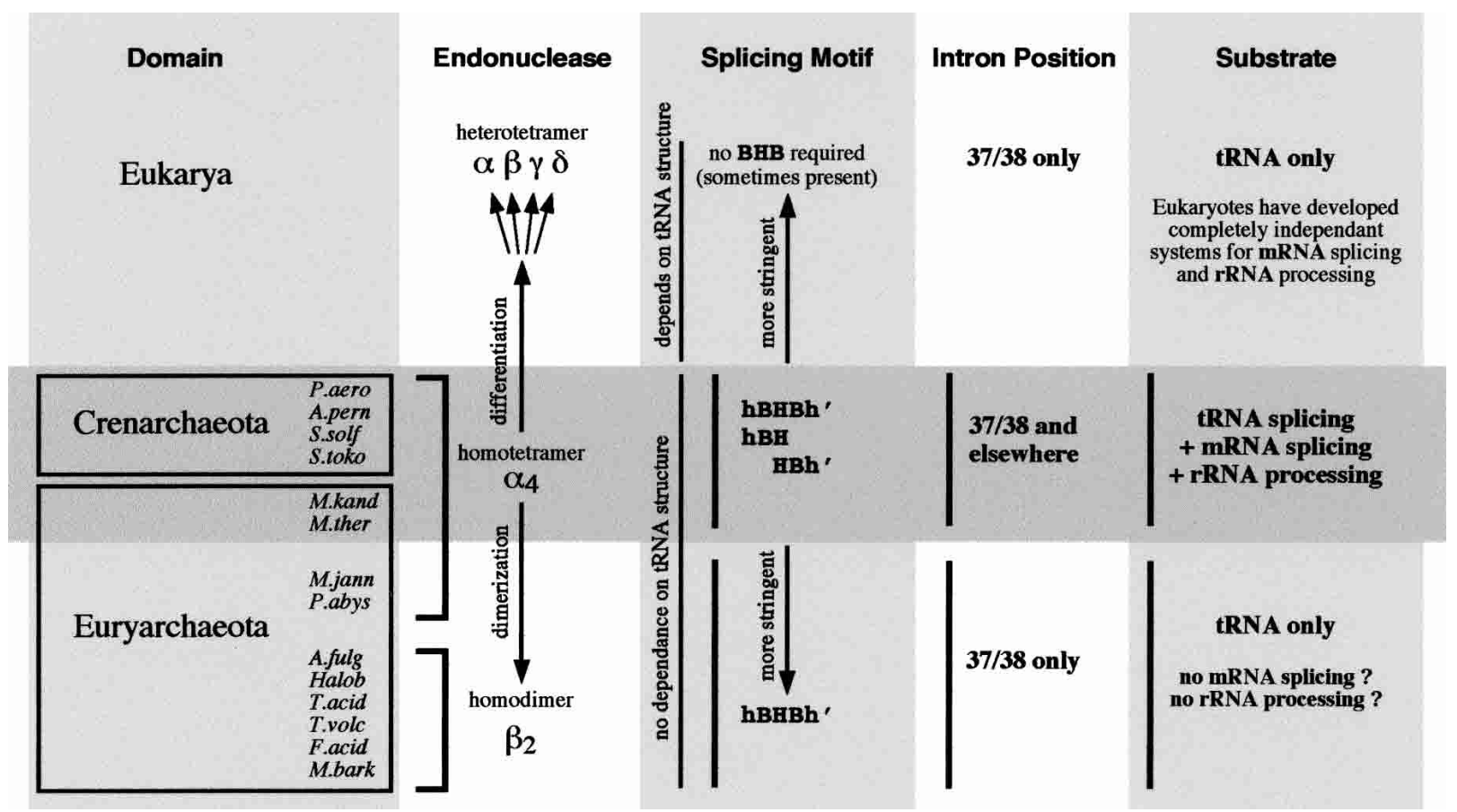

FIGURE 6. Endonuclease-based phylogeny of pre-tRNA introns splicing motifs and related features. The homotetrameric $\alpha_{4}$ endonuclease splicing at characteristic intron-exon junctions of archaeal pre-tRNAs (present in most Crenarchaeota and a few Euryarchaeota) is assumed to have evolved divergently towards a homodimeric $\beta_{2}$ enzyme in Euryarchaeota and into a heterotetrameric $\alpha, \beta, \delta, \gamma$ enzyme in Eukarya (see text). The monomer of the homotetrameric ancestral splicing endonuclease is designated by " $\alpha$ "; that of the homodimeric enzyme is designated by " $\beta$ " and the four different subunits of the eukaryotic heterotetrameric enzyme by " $\alpha, \beta, \gamma$, and $\delta$ ". Note that the size of the $\beta$ subunit is about twice that of the $\alpha$ subunit and probably results from gene duplication and subsequent fusion of an ancestral $\alpha$-like subunit gene (Lykke-Andersen and Garrett 1997; Bujnicki and Rychlewski 2000; Li and Abelson 2000).

have introns elsewhere than 37/38: T. pendens, a crenarchaeota, has an intron in the V-arm of its tDNA-Gly (CCC), but the nature of its splicing enzyme, homotetramer or homodimer, is not yet known (Kjems et al. 1989b).

\section{An ancient endonuclease from Crenarchaeota might be at the origin of the present-day endonucleases in the three biological domains}

The above considerations on the oligomeric structure diversity of endonucleases together with our intron sequence analyses led us to hypothesize that the more primitive homotetrameric $\alpha_{4}$ enzyme might tolerate less perfect $\mathbf{h B}$ $\mathbf{H B h}^{\prime}$ structures of the $\mathbf{h B H}$ or $\mathbf{H B h}^{\prime}$ types and have less stringent structural needs than its homodimeric $\left(\beta_{2}\right)$ successor, which requires more perfect $\mathbf{h B H B h}$ ' structures. Assuming that the endonuclease of Crenarchaeota still behaves as the enzyme that was present in the last universal common ancestor before the divergence into the three major forms of life (Archaea, Bacteria, and Eukarya), one can imagine the following scenario for the evolution and appearence of the different forms of the present-day endonucleases (Fig. 6). Evolution may have started from a homotretrameric $\alpha_{4}$-type of RNA splicing endonuclease. This primitive form of enzyme was probably able to splice intron-containing RNAs at a simple structural motif like the $\mathbf{h B H}$ or $\mathbf{B H h}$ ' located at any position of an RNA precursor. After divergence of Crenarchaeota to the Euryarchaeota kingdom, a more stringent homodimeric form of tRNAendonuclease $\left(\beta_{2}\right)$ appeared that might have resulted from gene duplication and fusion followed by mutations. This newly formed enzyme could have progressively evolved into a site-specific endonuclease that cleaves only pre-tRNAs in the anticodon loop at position 37/38. With the appearence of the Eukarya, the same primitive $\alpha_{4}$ precursor might have divergently evolved into an even more stringent and specialized heterotetrameric tRNA-endonucleases encompassing four different but related $\alpha, \beta, \gamma$, and $\delta$ subunits (Bujnicki and Rychlewski 2000). This enzyme would now cleave pre-tRNAs exclusively at position $37 / 38$ within a correct 3D folding of the tRNA molecule (see Di Nicola Negri et al. 1997; Trotta et al. 1997; for review, see Belfort and Weiner 1997; Trotta and Abelson 1999).

In support of this last hypothesis, it could well be that the archaeal relaxed $\mathbf{h B H}$ motif is an archaic form of the present day eukaryotic 3 ' splicing motif " $\mathrm{hBh}$ " (see above), in which the universal pyrimidine-32 in the anticodon loop must now pair with a conserved purine at position -3 from the $3^{\prime}$ side of the intron (the so-called "A-I" base pair; Baldi et al. 1992). It is also interesting to note that the eukaryal endonuclease has still retained its ability to splice the archaeal recognition signal $\left(\mathbf{h B H B h} \mathbf{h}^{\prime}\right.$, in fact $\mathbf{h B H}$ or $\mathbf{H B h}^{\prime}$ 
motif) within a tRNA ${ }^{\text {arch-euka }}$ whereas the purified endonuclease from the crenarchaeon S. solfataricus can also accurately splice the intron within the same chimeric pretRNA (Fabbri et al. 1998). Moreover, with certain chimeric pre-tRNAs, higher eukaryotic tRNA splicing endonucleases, such as the one from Xenopus laevis, can also cleave in a mode that is independent of the mature domain of the tRNA when substrates are able to form the perfect $\mathbf{h B H B h} \mathbf{H}^{\prime}$ splicing motif, the A-I base pair now playing a pivotal role in this process (Fruscoloni et al. 2001). Last but not least, from thorough analyses of tRNA populations in 60 fully sequenced genomes, Xue et al. (2003) recently argued that both Eukarya and Bacteria probably originated from the phylum Crenarchaeota, whereas the Euryarchaeota evolved from the same ancestor of Crenarchaeota, but independently of Eukarya and Bacteria. Our proposal concerning the independent evolution of the endonucleases found in the three biological domains fits well with this idea.

\section{MATERIALS AND METHODS}

\section{Search for introns in archaeal tDNAs}

Compilation of all intron sequences and their corresponding locations within $\mathrm{tDNA}$ was first performed by using automatic procedures (tRNA-ScanSE [Lowe and Eddy 1997] or cloverleaf tDNA search [Marck and Grosjean 2002]). This allowed us to easily identify all introns present at the usual position $37 / 38$ of the anticodon loop. Data from the literature (see references in Table 1), as well as those posted on "GtRDB: The genomic tRNA database" (http://rna.wustl.edu/GtRDB/) and the genome-specific websites (see GOLD website; http://igweb.integratedgenomics.com/GOLD/) were also taken into account. To locate introns at positions other than the canonical position 37/38 in archaeal tDNAs and missed by the above mentioned procedures, we specifically searched and visually inspected the cloverleaf fit of each individual tDNA that looked "abnormal" on the base of its size. The different criteria used to identify "abnormally located" introns in such "abnormally long" tDNAs were the following (see details in Marck and Grosjean 2002): (1) there exist rules that define the cloverleaf structure of archaeal tRNAs; (2) there is little or no tDNA redundancy in archaeal genomes; (3) a complete tDNA population able to translate all sense codons according to the known wobbling rules has to exist in every genome analyzed; and (4) like those located at position $37 / 38$, any putative spliceable introns located elesewhere than at position 37/38 should harbor a minimal BHB structure (the central $\mathrm{H}$ helix and at least one bulge; see Results). All introns located at unusual positions in tDNA are referred to as "noncanonical introns." A complete list of all intron sequences (canonical or noncanonical) and their exact locations within the tDNAs of the 18 archaea examined in this work is given as Supplementary Material.

\section{Endonuclease type determination}

Identification of the type of endonuclease, homotetrameric like in M. jannaschii (Li et al. 1998) or homodimeric like in A. fulgidus (Li and Abelson 2000), in each genome analyzed was performed by inspection of the size of the protein using a Blast search on Gen-
Bank (Altschul et al. 1997) with the M. jannaschii protein as entry. Homotetramer-type of endonucleases have a monomer size in the range $170-187$ residues (here referred to as the $\alpha$-type subunit), whereas homodimer-type endonucleases have a monomer size in the range $288-353$ residues (here referred to as the $\beta$-type subunit; see also Lykke-Andersen and Garrett 1997).

\section{Nomenclature}

We have redefined the canonical "BHB" splicing motif (Kjems et al. 1989a; Thompson and Daniels 1990; for review, see Trotta and Abelson 1999) that consists of a central 4-bp helix (H) flanked by

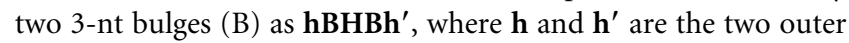
double helices closing the bulges. The BHB model does not specify the length of these helices, but it has been estimated, from a docking analysis of the NMR structure of a $\mathbf{h B H B h}$ ' RNA substrate (Diener and Moore 1998) onto the crystal structure of the $A$. fulgidus homodimeric endonuclease, that a minimal length of $2 \mathrm{bp}$ is required for the splicing to occur ( $\mathrm{Li}$ and Abelson 2000). We have retained the same minimal requirement and refer to the splicing motif as $\mathbf{h B H B h}$ ' if both $\mathbf{h}$ and $\mathbf{h}^{\prime}$ helices are at least $2 \mathrm{bp}$ long, whereas we refer to it as $\mathbf{h B H}$ or $\mathbf{H B h}^{\prime}$ if the $\mathbf{h}^{\prime}$ or $\mathbf{h}$ helix is shorter than $2 \mathrm{bp}$, respectively. The one-letter code used for nucleic acids consensus sequences (Cornish-Bowden 1985) is given in the legend to Figure 1.

\section{ACKNOWLEDGMENTS}

We thank Jean-Pierre Waller (Gif-sur-Yvette) and Carl Mann (SBGM, Saclay) for improving the manuscript and for useful critical comments. We thank Jean-Pierre Bachellerie (LBME, Toulouse) and Ramesh Gupta (Southern Illinois University, Carbondale) for helpful critical comments. We acknowledge Yves Boulard (SBGM, Saclay) for help in running tRNAscan-SE. We thank the DOE Joint Genome Institute for permission to use the genome data of the archaea Ferroplasma acidarmanus and Methanosarcina barkeri. The work of the DOE Joint Genome Institute was performed under the auspices of the U.S. Department of Energy, Office of Biological and Environmental Research, by the University of California, Lawrence Livermore National Laboratory under Contract No. W-7405-Eng-48, Lawrence Berkeley National Laboratory under contract No. DE-AC03-76SF00098, and Los Alamos National Laboratory under contract No. W-7405-ENG-36. This work was supported in part by the CNRS (Programme Interdépartemental Géomicrobiologie des Environnements Extrêmes, Géomex 2002-2003).

The publication costs of this article were defrayed in part by payment of page charges. This article must therefore be hereby marked "advertisement" in accordance with 18 USC section 1734 solely to indicate this fact.

Received July 17, 2003; accepted September 8, 2003.

\section{REFERENCES}

Altschul, S.F., Madden, T.L., Schaffer, A.A., Zhang, J., Zhang, Z., Miller, W., and Lipman, D.J. 1997. Gapped BLAST and PSIBLAST: A new generation of protein database search programs. Nucleic Acids Res. 25: 3389-3402.

Armbruster, D.W. and Daniels, C.J. 1997. Splicing of intron-contain- 
ing tRNATrp by the archaeon Haloferax volcanii occurs independent of mature tRNA structure. J. Biol. Chem. 272: 19758-19762.

Bachellerie, J.P., Cavaillé, J., and Huttenhofer, A. 2002. The expanding snoRNA world. Biochimie 84: 775-790.

Baldi, M.I., Mattoccia, E., Bufardeci, E., Fabbri, S., and TocchiniValentini, G.P. 1992. Participation of the intron in the reaction catalyzed by the Xenopus tRNA splicing endonuclease. Science 255: $1404-1408$.

Belfort, M. and Weiner, A. 1997. Another bridge between kingdoms: tRNA splicing in archaea and eukaryotes. Cell 89: 1003-1006.

Bujnicki, J.M. and Rychlewski, L. 2000. Prediction of a common fold for all four subunits of the yeast tRNA splicing endonuclease: Implications for the evolution of the EndA/Sen family. FEBS Lett. 486: 328-329.

Bult, C.J., White, O., Olsen, G.J., Zhou, L., Fleischmann, R.D., Sutton, G.G., Blake, J.A., FitzGerald, L.M., Clayton, R.A., Gocayne, J.D., et al. 1996. Complete genome sequence of the methanogenic archaeon, Methanococcus jannaschii. Science 273: 1058-1073.

Burggraf, S., Larsen, N., Woese, C.R., and Stetter, K.O. 1993. An intron within the $16 \mathrm{~S}$ ribosomal RNA gene of the archaeon Pyrobaculum aerophilum. Proc. Natl. Acad. Sci. 90: 2547-2550.

Cheong, C., Varani, G., and Tinoco Jr., I. 1990. Solution structure of unusually stable RNA hairpin. Nature 346: 680-682.

Ciammaruconi, A. and Londei, P. 2001. In vitro processing of the $16 \mathrm{~S}$ rRNA of the thermophilic archaeon Sulfolobus solfataricus. J. Bacteriol. 183: 3866-3874.

Cohen, G.N., Barbe, V., Flament, D., Galperin, M., Heilig, R., Lecompte, O., Poch, O., Prieur, D., Querellou, J., Ripp, R., et al. 2003. An integrated analysis of the genome of the hyperthermophilic archaeon Pyrococcus abyssi. Mol. Microbiol. 47: 1495-1512.

Constantinesco, F., Motorin, Y., and Grosjean, H. 1999. Transfer RNA modification enzymes from Pyrococcus furiosus: Detection of the enzymatic activities in vitro. Nucleic Acids Res. 27: 1308-1315.

Cornish-Bowden, A. 1985. Nomenclature for incompletely specified bases in nucleic acid sequences: Recommendations 1984. Nucleic Acids Res. 13: 3021-3030.

Culbertson, M.R. and Winey, M. 1989. Split tRNA genes and their products: A paradigm for the study of cell function and evolution. Yeast 5: 405-427.

Dalgaard, J.Z. and Garrett, R.A. 1992. Protein-coding introns from the $23 \mathrm{~S}$ rRNA-encoding gene form stable circles in the hyperthermophilic archaeon Pyrobaculum organotrophum. Gene 121: 103-110.

Daniels, C.J. and Dougas, S.E. 1986. Genes for transfer RNAs in $\mathrm{Ha}$ lobacterium volcanii. System. Appl. Microbiol. 7: 26-29.

Daniels, C.J., Gupta, R., and Doolittle, W.F. 1985. Transcription and excision of a large intron in the tRNATrp gene of an archaebacterium, Halobacterium volcanii. J. Biol. Chem. 260: 3132-3134.

Datta, P.K., Hawkins, L.K., and Gupta, R. 1989. Presence of an intron in elongator methionine-tRNA of Halobacterium volcanii. Can. J. Microbiol. 35: 189-194.

Dennis, P.P., Ziesche, S., and Mylvaganam, S. 1998. Transcription analysis of two disparate rRNA operons in the halophilic archaeon Haloarcula marismortui. J. Bacteriol. 180: 4804-4813.

Dennis, P.P., Omer, A., and Lowe, T. 2001. A guided tour: Small RNA function in Archaea. Mol. Microbiol. 40: 509-519.

Deppenmeier, U., Johann, A., Hartsch, T., Merkl, R., Schmitz, R.A., Martinez-Arias, R., Henne, A., Wiezer, A., Baumer, S., Jacobi, C., et al. 2002. The genome of Methanosarcina mazei: Evidence for lateral gene transfer between bacteria and archaea. J. Mol. Microbiol. Biotechnol. 4: 453-461.

Di Nicola Negri, E., Fabbri, S., Bufardeci, E., Baldi, M.I., Gandini Attardi, D., Mattoccia, E., and Tocchini-Valentini, G.P. 1997. The eucaryal tRNA splicing endonuclease recognizes a tripartite set of RNA elements. Cell 89: 859-866.

Diener, J.L. and Moore, P.B. 1998. Solution structure of a substrate for the archaeal pre-tRNA splicing endonucleases: The bulge-helixbulge motif. Mol. Cell 1: 883-894.

d'Orval, B.C., Bortolin, M.L., Gaspin, C., and Bachellerie, J.P. 2001. Box C/D RNA guides for the ribose methylation of archaeal tRNAs.
The tRNA-Trp intron guides the formation of two ribose-methylated nucleosides in the mature tRNA-Trp. Nucleic Acids Res. 29: 4518-4529.

Durovic, P. and Dennis, P.P. 1994. Separate pathways for excision and processing of $16 \mathrm{~S}$ and $23 \mathrm{~S}$ rRNA from the primary rRNA operon transcript from the hyperthermophilic archaebacterium Sulfolobus acidocaldarius: Similarities to eukaryotic rRNA processing. Mol. Microbiol. 13: 229-242.

Fabbri, S., Fruscoloni, P., Bufardeci, E., Di Nicola Negri, E., Baldi, M.I., Attardi, D.G., Mattoccia, E., and Tocchini-Valentini, G.P. 1998. Conservation of substrate recognition mechanisms by tRNA splicing endonucleases. Science 280: 284-286.

Fitz-Gibbon, S.T., Ladner, H., Kim, U.J., Stetter, K.O., Simon, M.I., and Miller, J.H. 2002. Genome sequence of the hyperthermophilic crenarchaeon Pyrobaculum aerophilum. Proc. Natl. Acad. Sci. 99: 984-989.

Fruscoloni, P., Baldi, M.I., and Tocchini-Valentini, G.P. 2001. Cleavage of non-tRNA substrates by eukaryal tRNA splicing endonucleases. EMBO Rep. 2: 217-221.

Galagan, J.E., Nusbaum, C., Roy, A., Endrizzi, M.G., Macdonald, P., FitzHugh, W., Calvo, S., Engels, R., Smirnov, S., Atnoor, D., et al. 2002. The genome of $M$. acetivorans reveals extensive metabolic and physiological diversity. Genome Res. 12: 532-542.

Garrett, R.A., Dalgaard, J., Larsen, N., Kjems, J., and Mankin, A.S. 1991. Archaeal rRNA operons. Trends Biochem. Sci. 16: 22-26.

Gaspin, C., Cavaillé, J., Erauso, G., and Bachellerie, J.P. 2000. Archaeal homologs of eukaryotic methylation guide small nucleolar RNAs: Lessons from the Pyrococcus genomes. J. Mol. Biol. 297: 895-906.

Grosjean, H., Constantinesco, F., Foiret, D., and Benachenhou, N. 1995. A novel enzymatic pathway leading to 1-methylinosine modification in Haloferax volcanii tRNA. Nucleic Acids Res. 23: $4312-4319$.

Grosjean, H., Szweykowska-Kulinska, Z., Motorin, Y., Fasiolo, F., and Simos, G. 1997. Intron-dependent enzymatic formation of modified nucleosides in eukaryotic tRNAs: A review. Biochimie 79: 293-302.

Hall, T.A. and Brown, J.W. 2001. The ribonuclease P family. Methods Enzymol. 341: 56-77.

Heus, H.A. and Pardi, A. 1991. Structural features that give rise to the unusual stability of RNA hairpins containing GNRA loops. Science 253: 191-194.

Itoh, T., Suzuki, K., and Nakase, T. 1998. Occurrence of introns in the $16 \mathrm{~S}$ rRNA genes of members of the genus Thermoproteus. Arch. Microbiol. 170: 155-161.

Kaine, B.P. 1987. Intron-containing tRNA genes of Sulfolobus solfataricus. J. Mol. Evol. 25: 248-254.

Kaine, B.P., Gupta, R., and Woese, C.R. 1983. Putative introns in tRNA genes of prokaryotes. Proc. Natl. Acad. Sci. USA 80: 3309-3312.

Kawarabayasi, Y., Sawada, M., Horikawa, H., Haikawa, Y., Hino, Y., Yamamoto, S., Sekine, M., Baba, S., Kosugi, H., Hosoyama, A., et al. 1998. Complete sequence and gene organization of the genome of a hyper-thermophilic archaebacterium, Pyrococcus horikoshii OT3. DNA Res. 5: 55-76.

Kawarabayasi, Y., Hino, Y., Horikawa, H., Yamazaki, S., Haikawa, Y., Jin-no, K., Takahashi, M., Sekine, M., Baba, S., Ankai, A., et al. 1999. Complete genome sequence of an aerobic hyper-thermophilic crenarchaeon, Aeropyrum pernix K1. DNA Res. 6: 83-101, 145-152.

Kawarabayasi, Y., Hino, Y., Horikawa, H., Jin-no, K., Takahashi, M., Sekine, M., Baba, S., Ankai, A., Kosugi, H., Hosoyama, A., et al. 2001. Complete genome sequence of an aerobic thermoacidophilic crenarchaeon, Sulfolobus tokodaii strain7. DNA Res. 8: 123-140.

Kawashima, T., Amano, N., Koike, H., Makino, S., Higuchi, S., Kawashima-Ohya, Y., Watanabe, K., Yamazaki, M., Kanehori, K., Kawamoto, T., et al. 2000. Archaeal adaptation to higher temperatures revealed by genomic sequence of Thermoplasma volcanium. Proc. Natl. Acad. Sci. 97: 14257-14262.

Kjems, J. and Garrett, R.A. 1988. Novel splicing mechanism for the ribosomal RNA intron in the archaebacterium Desulfurococcus mobilis. Cell 54: 693-703. 
1991. Ribosomal RNA introns in archaea and evidence for RNA conformational changes associated with splicing. Proc. Natl. Acad. Sci. 88: 439-443.

Kjems, J., Jensen, J., Olesen, T., and Garrett, R.A. 1989a. Comparison of transfer RNA and ribosomal RNA intron splicing in the extreme thermophile and archaebacterium Desulfurococcus mobilis. Can. J. Microbiol. 35: 210-214.

Kjems, J., Leffers, H., Olesen, T., and Garrett, R.A. 1989b. A unique tRNA intron in the variable loop of the extreme thermophile Thermofilum pendens and its possible evolutionary implications. J. Biol. Chem. 264: 17834-17837.

Kleman-Leyer, K., Armbruster, D.W., and Daniels, C.J. 1997. Properties of $H$. volcanii tRNA intron endonuclease reveal a relationship between the archaeal and eucaryal tRNA intron processing systems. Cell 89: 839-847.

Klenk, H.P., Clayton, R.A., Tomb, J.F., White, O., Nelson, K.E., Ketchum, K.A., Dodson, R.J., Gwinn, M., Hickey, E.K., Peterson, J.D., et al. 1997. The complete genome sequence of the hyperthermophilic, sulphate-reducing archaeon Archaeglobus fulgidus. Nature 390: $364-370$

Krasilnikov, A.S., Yang, X., Pan, T., and Mondragòn, A. 2003. Crystal structure of the specificity domain of Ribonuclease P. Nature 421: 760-764.

Lavrov, D.V., Brown, W.M., and Boore, J.L. 2000. A novel type of RNA editing occurs in the mitochondrial tRNAs of the centipede Lithobius forficatus. Proc. Natl. Acad. Sci. 97: 13738-13742.

Lee, M.C. and Knapp, G. 1985. Transfer RNA splicing in Saccharomyces cerevisiae. Secondary and tertiary structures of the substrates. J. Biol. Chem. 260: 3108-3115.

Li, H. and Abelson, J. 2000. Crystal structure of a dimeric archaeal splicing endonuclease. J. Mol. Biol. 302: 639-648.

Li, H., Trotta, C.R., and Abelson, J. 1998. Crystal structure and evolution of a transfer RNA splicing enzyme. Science 280: 279-284.

Lowe, T.M. and Eddy, S.R. 1997. tRNAscan-SE: A program for improved detection of transfer RNA genes in genomic sequence. Nucleic Acids Res. 25: 955-964.

Lykke-Andersen, J. and Garrett, R.A. 1994. Structural characteristics of the stable RNA introns of archaeal hyperthermophiles and their splicing junctions. J. Mol. Biol. 243: 846-855.

- 1997. RNA-protein interactions of an archaeal homotetrameric splicing endoribonuclease with an exceptional evolutionary history. EMBO J. 16: 6290-6300.

Lykke-Andersen, J., Aagaard, C., Semionenkov, M., and Garrett, R.A. 1997. Archaeal introns: Splicing, intercellular mobility and evolution. Trends Biochem. Sci. 22: 326-331.

Marck, C. and Grosjean, H. 2002. tRNomics: Analysis of tRNA genes from 50 genomes of Eukarya, Archaea, and Bacteria reveals anticodon-sparing strategies and domain-specific features. RNA 8: 1189-1232.

Michel, F. and Costa, M. 1998. Inferring RNA structures by phylogenetic and genetic analyses. In RNA structure and function (eds. R.W. Simons and M. Grunberg-Manago), pp. 695-726. pp175202. Cold Spring Harbor Laboratory Press, Cold Spring Harbor, NY.

Mörl, M. and Marchfelder, A. 2001. The final cut. The importance of tRNA 3'-processing. EMBO Rep. 2: 17-20.

Ng, W.V., Kennedy, S.P., Mahairas, G.G., Berquist, B., Pan, M., Shukla, H.D., Lasky, S.R., Baliga, N.S., Thorsson, V., Sbrogna, J., et al. 2000. Genome sequence of Halobacterium species NRC-1. Proc. Natl. Acad. Sci. 97: 12176-12181.

Nomura, N., Sako, Y., and Uchida, A. 1998. Molecular characterization and postsplicing fate of three introns within the single rRNA operon of the hyperthermophilic archaeon Aeropyrum pernix K1. J. Bacteriol. 180: 3635-3643.

Omer, A.D., Ziesche, S., Decatur, W.A., Fournier, M.J., and Dennis, P.P. 2003. RNA-modifying machines in archaea. Mol. Microbiol. 48: 617-629.

Pintard, L., Lecointe, F., Bujnicki, J.M., Bonnerot, C., Grosjean, H., and Lapeyre, B. 2002. Trm7p catalyses the formation of two 2'$O$-methylriboses in yeast tRNA anticodon loop. EMBO J. 21:
$1811-1820$.

Robb, F.T., Maeder, D.L., Brown, J.R., DiRuggiero, J., Stump, M.D., Yeh, R.K., Weiss, R.B., and Dunn, D.M. 2001. Genomic sequence of hyperthermophile, Pyrococcus furiosus: Implications for physiology and enzymology. Methods Enzymol. 330: 134-157.

Ruepp, A., Graml, W., Santos-Martinez, M.L., Koretke, K.K., Volker, C., Mewes, H.W., Frishman, D., Stocker, S., Lupas, A.N., and Baumeister, W. 2000. The genome sequence of the thermoacidophilic scavenger Thermoplasma acidophilum. Nature 407: 508-513.

Salgia, S.R., Singh, S.K., Gurha, P., and Gupta, R. 2003. Two reactions of Haloferax volcanii RNA splicing enzymes: Joining of exons and circularization of introns. RNA 9: 319-330.

She, Q., Singh, R.K., Confalonieri, F., Zivanovic, Y., Allard, G., Awayez, M.J., Chan-Weiher, C.C., Clausen, I.G., Curtis, B.A., De Moors, A., et al. 2001. The complete genome of the crenarchaeon Sulfolobus solfataricus P2. Proc. Natl. Acad. Sci. 26: 7835-7940.

Slesarev, A.I., Mezhevaya, K.V., Makarova, K.S., Polushin, N.N., Shcherbinina, O.V., Shakhova, V.V., Belova, G.I., Aravind, L., Natale, D.A., Rogozin, I.B., et al. 2002. The complete genome of hyperthermophile Methanopyrus kandleri AV19 and monophyly of archaeal methanogens. Proc. Natl. Acad. Sci. 99: 4644-4649.

Smith, D.R., Doucette-Stamm, L.A., Deloughery, C., Lee, H., Dubois, J., Aldredge, T., Bashirzadeh, R., Blakely, D., Cook, R., Gilbert, K., et al. 1997. Complete genome sequence of Methanobacterium thermoautotrophicum DH: Functional analysis and comparative genomics. J. Bacteriol. 179: 7135-7155.

Sprinzl, M., Horn, C., Brown, M., Ioudovitch, A., and Steinberg, S. 1998. Compilation of tRNA sequences and sequences of tRNA genes. Nucleic Acids Res. 26: 148-153.

Swerdlow, H. and Guthrie, C. 1984. Structure of intron-containing tRNA precursors. Analysis of solution conformation using chemical and enzymatic probes. J. Biol. Chem. 259: 5197-5207.

Tang, T.H., Rozhdestvensky, T.S., d'Orval, B.C., Bortolin, M.L., Huber, H., Charpentier, B., Branlant, C., and Bachellerie, J.P., Brosius, J., and Huttenhofer, A. 2002. RNomics in Archaea reveals a further link between splicing of archaeal introns and rRNA processing. Nucleic Acids Res. 30: 921-930.

Thomm, M. and Wich, G. 1988. An archaebacterial promoter element for stable RNA genes with homology to the TATA box of higher eukaryotes. Nucleic Acids Res. 16: 151-163.

Thompson, L.D. and Daniels, C.J. 1988. A tRNA(Trp) intron endonuclease from Halobacterium volcanii. Unique substrate recognition properties. J. Biol. Chem. 263: 17951-17959.

. 1990. Recognition of exon-intron boundaries by the Halobacterium volcanii tRNA intron endonuclease. J. Biol. Chem. 265: 18104-18111.

Thompson, L.D, Brandon, L.D., Nieuwlandt, D.T., and Daniels, C.J. 1989. Transfer RNA intron processing in the halophilic archaebacteria. Can. J. Microbiol. 35: 36-42.

Trotta, C.R. and Abelson, J. 1999. tRNA splicing: An RNA world add-on or an ancient reaction? In The RNA world, 2nd ed. (eds. R.F. Gestel et al.), pp. 561-584. Cold Spring Harbor Laboratory Press, Cold Spring Harbor, NY.

Trotta, C.R., Miao, F., Arn, E.A., Stevens, S.W., Ho, C.K., Rauhut, R., and Abelson, J.N. 1997. The yeast tRNA splicing endonuclease: A tetrameric enzyme with two active site subunits homologous to the archaeal tRNA endonucleases. Cell 89: 849-858.

Watanabe, Y., Yokobori, S., Inaba, T., Yamagishi, A., Oshima, T., Kawarabayasi, Y., Kikuchi, H., and Kita, K. 2002. Introns in protein-coding genes in Archaea. FEBS Lett. 510: 27-30.

Wich, G., Leinfelder, W., and Böck, A. 1987. Genes for stable RNA in the extreme thermophile Thermoproteus tenax: introns and transcription signal. EMBO J. 6: 523-528.

Xue, H., Tong, K.L., Marck, C., Grosjean, H., and Wong, J.T. 2003. Transfer RNA paralogs: Evidence for genetic code-amino acid biosynthesis coevolution and an archaeal root of life. Gene 310: 59-66.

Zahler, N.H., Christian, E.L., and Harris, M.E. 2003. Recognition of the $5^{\prime}$ leader of pre-tRNA substrates by the active site of ribonuclease P. RNA 9: 734-745. 

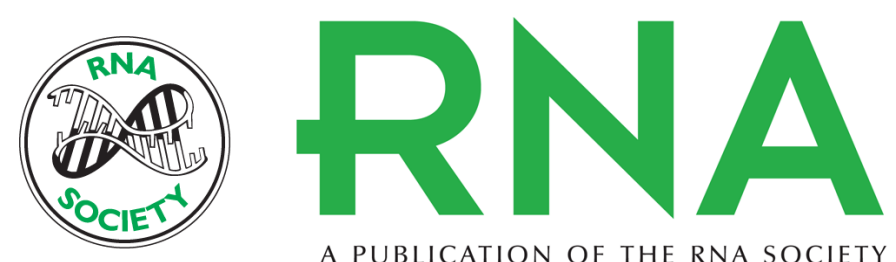

A PUBLICATION OF THE RNA SOCIETY

\section{Identification of BHB splicing motifs in intron-containing tRNAs from 18 archaea: evolutionary implications}

CHRISTIAN MARCK and HENRI GROSJEAN

RNA 2003 9: 1516-1531

References This article cites 79 articles, 31 of which can be accessed free at:

http://rnajournal.cshlp.org/content/9/12/1516.full.html\#ref-list-1

License

Email Alerting Receive free email alerts when new articles cite this article - sign up in the box at the Service top right corner of the article or click here.

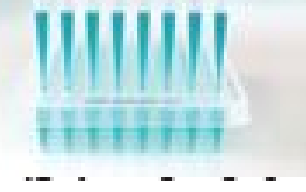

Providing Precise Solutions for your research.

To subscribe to $R N A$ go to:

http://rnajournal.cshlp.org/subscriptions 